\title{
Scalable and Parallel Tweezer Gates for Quantum Computing with Long Ion Strings
}

\author{
Tobias Olsacher®,,${ }^{1,2}{ }^{*}$ Lukas Postler, ${ }^{3}$ Philipp Schindler®, ${ }^{3}$ Thomas Monz, ${ }^{3}$ Peter Zoller $\odot,{ }^{1,2}$ and \\ Lukas M. Sieberer@1, $1,{ }^{1, \dagger}$ \\ ${ }^{1}$ Center for Quantum Physics University of Innsbruck, Innsbruck, Austria \\ ${ }^{2}$ Institute for Quantum Optics and Quantum Information of the Austrian Academy of Sciences, Innsbruck, Austria \\ ${ }^{3}$ Institut für Experimentalphysik, Universität Innsbruck, Innsbruck, Austria
}

(Received 26 August 2020; accepted 4 November 2020; published 2 December 2020)

\begin{abstract}
Trapped-ion quantum computers have demonstrated high-performance gate operations in registers of about ten qubits. However, scaling up and parallelizing quantum computations with long one-dimensional (1D) ion strings is an outstanding challenge due to the global nature of the motional modes of the ions, which mediate qubit-qubit couplings. Here, we devise methods to implement scalable and parallel entangling gates by using engineered localized phonon modes. We propose to tailor such localized modes by tuning the local potential of individual ions with programmable optical tweezers. Localized modes of small subsets of qubits form the basis to perform entangling gates on these subsets in parallel. We demonstrate the inherent scalability of this approach by presenting analytical and numerical results for long 1D ion chains and even for infinite chains of uniformly spaced ions. Furthermore, we show that combining our methods with optimal coherent control techniques allows realization of maximally dense universal parallelized quantum circuits.
\end{abstract}

DOI: 10.1103/PRXQuantum.1.020316

\section{INTRODUCTION}

Trapped atomic ions are a leading platform for quantuminformation processing [1-9]. High-fidelity gate operations, qubit initialization and readout, as well as long coherence times have been demonstrated in trapped-ion quantum computers, which consist of tens of individually addressable qubits [10-14]. However, while state-of-theart ion traps can sustain one-dimensional (1D) chains of more than a hundred ions, scaling up and, in particular, parallelizing gate operations is challenging due to the increasing complexity of the spectrum of phonon modes, which serve as data buses for the transmission of quantum information $[1,15]$.

The spectrum of phonon modes becomes dense for long ion crystals [16,17], and, consequently, gate operations that rely on using a single phonon mode become slow due to the necessity to spectrally resolve this mode. In contrast, implementing gates that use multiple modes, requires

\footnotetext{
*tobias.olsacher@uibk.ac.at

†lukas.sieberer@uibk.ac.at
}

Published by the American Physical Society under the terms of the Creative Commons Attribution 4.0 International license. Further distribution of this work must maintain attribution to the author(s) and the published article's title, journal citation, and DOI. solving optimal control problems [18-26], which become exceedingly complex, in particular, when gates should be performed on many qubits in parallel $[23,24]$. This additional complication to implement parallel gates is due to the collective and thus nonlocal nature of phonon modes, which stands in contrast to the goal of effecting two-qubit entangling operations that locally address individual pairs of ions.

An alternative approach is provided by segmented traps, in which small ion crystals are shuttled between different zones that are dedicated to qubit storage and manipulation, respectively [27]. Shuttling operations are comparatively time consuming, and form a bottleneck for fast quantum computations. This disadvantage is mitigated by the fact that in traps with several interaction zones, gates can be performed in parallel. Although the fabrication and operation of segmented traps is technologically challenging, quantum-information processing, which relies heavily on ion shuttling, has been demonstrated experimentally [28-30].

In this paper, we address the challenge of scaling up and parallelizing quantum computations with long 1D ion strings by combining well-developed conventional linear Paul traps [31] with programmable optical tweezer arrays, which are commonly utilized as a powerful tool in quantum simulation with neutral Rydberg atoms [32,33]. Here, instead, we consider using programmable optical tweezer 
(a)

(b)

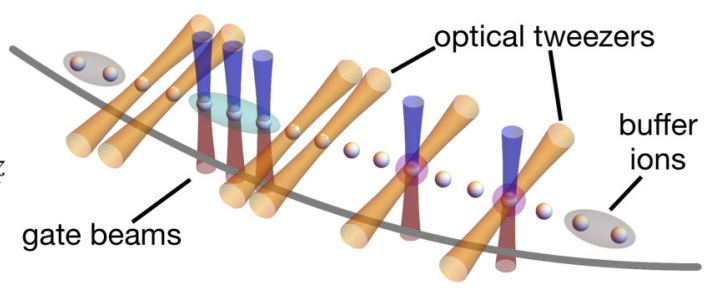

(b)

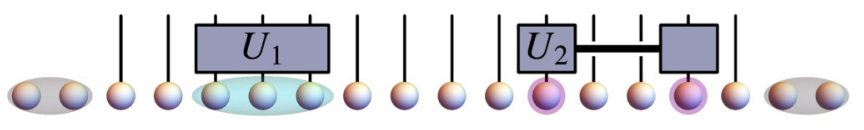

(c)

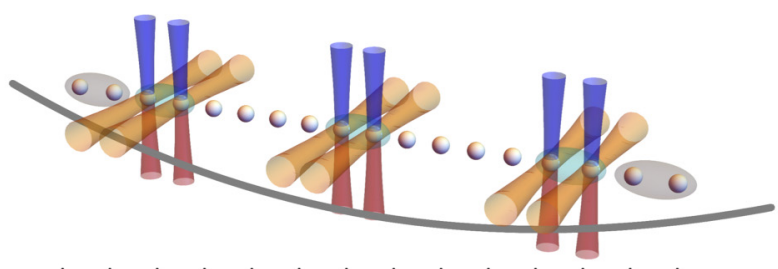

(d)

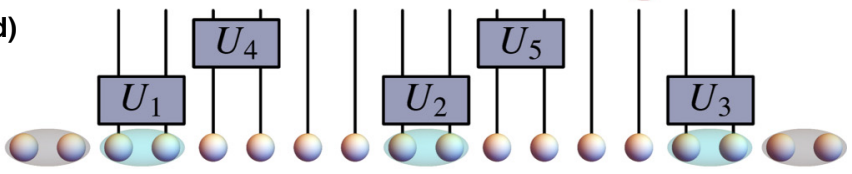

FIG. 1. Schematic setup and circuits. (a) Specific ions in a long 1D chain are pinned by optical tweezers. This leads to the formation of localized phonon modes, e.g., for the subregister formed by the three ions that are marked by cyan shading, and for the pair of distant qubits that are marked by pink shading and are pinned by equally strong optical potentials. (b) The localized phonon modes can be utilized to implement multiqubit entangling gates. Buffer ions at the ends of the chain are not used as qubits. Addressed lasers, which are required to perform entangling gates, are indicated schematically in (a) as blue and red beams. (c) In this work, we focus on implementing parallel two-qubit entangling gates, which use the localized phonon modes of pairs of neighboring pinned ions. (d) After a first layer of the circuit, which corresponds to the product of gates $U_{1} U_{2} U_{3}$ that act on distinct qubits, the tweezers are focused on different ions to perform the second layer corresponding to the gate operation $U_{4} U_{5}$.

arrays to pin specific ions in a linear trap [34], and thereby engineer localized phonon modes [35-37]. These designer phonon modes form the basis to implement scalable and parallel entangling gates. The required capabilities to optically address individual ions are available in current experiments [38,39]. By dynamically reconfiguring the tweezer array, the designer phonon modes can be adjusted during running quantum computations, and thus offer great flexibility to achieve an effective "optical segmentation" of the ion chain.

Possible quantum circuits provided by optical segmentation are illustrated schematically in Figs. 1(a) and 1(b): in the first example, a subregister formed by three consecutive qubits, which are marked by cyan shading, is decoupled from the other qubits by "optical tweezer walls" [34] that consist of pairs of optically pinned ions. By using the phonon modes that are localized in between the tweezer walls, a multiqubit gate can be performed on the subregister. The second example relies on the long-range connectivity in chains of trapped ions to implement an entangling gate between the two qubits marked by pink shading and which are both pinned by optical tweezers.

In this work, we focus on the implementation of quantum circuits, which are composed of parallel gates between pairs of nearest-neighboring qubits. Pinning pairs of neighboring ions with optical tweezers, as illustrated in Fig. 1(c), gives rise to localized phonon modes, which correspond to center-of-mass (COM) and stretch oscillations of the pinned pairs. In microtraps with segmented electrodes, an analogous local mode structure arises for two ions, which are shuttled to an interaction zone of the trap. The optical segmentation enables performing entangling gates on all pairs of pinned ions in parallel, which corresponds to the first layer of the quantum circuit shown in Fig. 1(d).

Due to the emergence of a local phonon-mode structure with only two relevant localized phonon modes per pinned pair, high-fidelity scalable and parallel gates can be performed in long ion chains without resorting to optimal coherent control techniques [18-26]. Further, the gate duration is determined by the splitting of the localized phonon modes, and is thus independent of the total number of ions in the chain. Indeed, as we show analytically and numerically, tweezer gates can be performed even in infinitely long chains [23].

Finally, we discuss different ways to minimize crosstalk between parallel two-qubit entangling gates based on optimal control of time-modulated laser-pulse amplitudes as developed by Duan et al. [17]. This enables the implementation of dense "brick-wall circuits." Such dense circuits have a wide range of applications, for example, in digital quantum simulation [40-42] of spin models with local interactions, or to realize random circuits [43], which are models for strongly chaotic quantum dynamics [44-48].

The paper is structured as follows: we start in Sec. II with a short review of some aspects of quantum gates with trapped ions. In Sec. III, we discuss how to design localized phonon modes for quantum computing. Subsequently, in Secs. IV and V, we show how these localized modes can be used to implement scalable and parallel tweezer gates with and without optimal control, respectively. We give an outlook in Sec. VI.

\section{ENTANGLING QUANTUM GATES WITH TRAPPED IONS}

For reference, we find it convenient to summarize some fundamentals of quantum computing with trapped ions. Based on this, we state the decomposition of quantum circuits into single-qubit and nearest-neighbor entangling gates and discuss how one can quantify the performance of a two-qubit quantum gate. 


\section{A. Quantum computing with trapped ions}

We consider an implementation of quantum logic gates in 1D chains of laser-cooled trapped ions that relies on the laser-induced coupling between long-lived internal states of the ions, which encode the qubits, and the phonon modes of the ion chain, which serve as quantum data buses. Entanglement between qubits is established through the exchange of real [1] or virtual [49-52] phonons. The latter approach is realized by geometric phase gates, which offer the advantage of being insensitive to finite temperatures of the phonon modes. We employ a particular type of geometric phase gate, which is known as the Mølmer-Sørensen gate [51]. In a suitable rotating frame, the corresponding qubit-phonon coupling Hamiltonian for $N$ ions with $3 N$ motional modes reads

$$
\begin{aligned}
H= & \sum_{\alpha \in\{x, y, z\}} \sum_{i, n=1}^{N} \eta_{\alpha, i}^{n} \hbar \Omega_{i}(t) \sin \left(\mu_{i} t\right) \\
& \times\left(a_{\alpha, n}^{\dagger} e^{i \omega_{\alpha, n} t}+\text { H.c. }\right) \sigma_{i}^{x},
\end{aligned}
$$

where the first sum is over the spatial directions $\alpha$ of the three-dimensional (3D) motion of the ions. As illustrated in Fig. 1(a), we choose the coordinate system such that $x$ and $y$ directions are transverse to the weak trap axis, which is along the $z$ direction. In the form given above, the Hamiltonian is valid in the Lamb-Dicke limit, in which the amplitudes of oscillations of the ions around their equilibrium positions are small in comparison to optical wavelengths. This justifies an expansion to first order in the Lamb-Dicke parameter matrix, which we define as

$$
\eta_{\alpha, i}^{n}=k_{L, \alpha} \sqrt{\frac{\hbar}{2 m \omega_{\alpha, n}}} M_{\alpha, i}^{n},
$$

with the effective laser wave vector with components $k_{L, \alpha}$ that are assumed to be equal for all ions [22]. Here $m$ is the mass of a single ion, $\omega_{\alpha, n}$ is the frequency of the phonon mode $n \in\{1, \ldots, N\}$ in spatial direction $\alpha$, and the element $M_{\alpha, i}^{n}$ of the phonon-mode matrix is given by the amplitude of the phonon-mode vector $n$ on ion $i \in\{1, \ldots, N\}$. The phonon-mode matrix is derived in Appendix A.

Hamiltonian (1) results from addressing the ions with pairs of laser beams, which are detuned by $\pm \mu_{i}$ from the qubit transition [49-52]. We assume that the detuning $\mu_{i}$ and the laser pulse shape as described by a time-modulated Rabi frequency $\Omega_{i}(t)$ can be chosen for each ion $i$ individually. The annihilation and creation operators of phonons with frequency $\omega_{\alpha, n}$ are denoted by $a_{\alpha, n}$ and $a_{\alpha, n}^{\dagger}$, respectively, and $\sigma_{i}^{x}$ is the Pauli matrix, which acts in the Hilbert space of the qubit that is encoded in ion $i$. We assume in the following that the lasers dominantly excite transverse $x$ modes, which is justified if the detuning from the excited transverse $x$ modes is much smaller than the frequency difference between the transverse $x$ modes and the transverse $y$ and longitudinal $z$ modes, respectively [17]. To simplify the notation, from now on we mostly omit the subscript $\alpha=x$.

The time evolution that is generated by the timedependent qubit-phonon Hamiltonian (1) is described by the unitary $[17,18,22,53-55]$

$$
U=\exp \left(\sum_{i=1}^{N} \phi_{i} \sigma_{i}^{x}+i \sum_{i<i^{\prime}=1}^{N} \chi_{i, i^{\prime}} \sigma_{i}^{x} \sigma_{i^{\prime}}^{x}\right) .
$$

The first term in the exponent with the operator

$$
\phi_{i}=\sum_{n=1}^{N}\left(\alpha_{i}^{n} a_{n}^{\dagger}+\text { H.c. }\right),
$$

induces qubit-state-dependent displacements of the phonon modes where

$$
\alpha_{i}^{n}=-i \eta_{i}^{n} \int_{0}^{\tau} d t \Omega_{i}(t) \sin \left(\mu_{i} t\right) e^{i \omega_{n} t} .
$$

If $\phi_{i} \neq 0$ for a given qubit $i$, unwanted entanglement is created between that qubit and the phonon modes. This can be prevented by carefully choosing the detuning and gate duration depending on the phonon spectrum. The desired qubit-qubit coupling term is given by

$$
\begin{aligned}
\chi_{i, i^{\prime}}= & \sum_{n=1}^{N} \eta_{i}^{n} \eta_{i^{\prime}}^{n} \int_{0}^{\tau} d t \int_{0}^{t} d t^{\prime} \sin \left[\omega_{n}\left(t-t^{\prime}\right)\right] \\
& \times\left[\Omega_{i}(t) \Omega_{i^{\prime}}\left(t^{\prime}\right) \sin \left(\mu_{i} t\right) \sin \left(\mu_{i^{\prime}} t^{\prime}\right)\right. \\
& \left.+\Omega_{i}\left(t^{\prime}\right) \Omega_{i^{\prime}}(t) \sin \left(\mu_{i} t^{\prime}\right) \sin \left(\mu_{i^{\prime}} t\right)\right] .
\end{aligned}
$$

We focus in the following on the implementation, based on Eq. (3), of quantum circuits with entangling operations between only neighboring qubits as illustrated in Fig. 1(d). Such circuits can be arranged in consecutive layers where gates within each layer are executed in parallel. A single layer can be written as

$$
U_{0}=\prod_{\left(i, i^{\prime}\right) \in I} U_{i, i^{\prime}}
$$

where $I$ is the set of all pairs of neighboring qubits on which gates are to be performed in the layer under consideration. Since any entangling two-qubit gate can be decomposed into single-qubit rotations and entangling operations, which are generated by $\sigma_{i}^{x} \sigma_{i^{\prime}}^{x}$ [56], we can assume without loss of generality that $U_{i, i^{\prime}}$ is an $x x$ gate,

$$
U_{i, i^{\prime}}=e^{i \chi_{i, i^{\prime}}^{0} \sigma_{i}^{x} \sigma_{i^{\prime}}^{x}}
$$

with couplings $\chi_{i, i^{\prime}}^{0}$ for different pairs $\left(i, i^{\prime}\right)$ all having the same positive or negative sign and $\left|\chi_{i, i^{\prime}}^{0}\right| \in[0, \pi / 4]$ [56]. 
Throughout this work we consider maximally entangling gates with $\chi_{i, i^{\prime}}^{0}= \pm \pi / 4$ as a benchmark case. This benchmark is particularly relevant since the unitary, Eq. (8), with $\chi_{i, i^{\prime}}^{0}= \pm \pi / 4$ is up to single-qubit rotations equivalent to the CNOT gate [21], which forms a universal gate set together with single-qubit rotations [57].

\section{B. Gate imperfections}

Any experimental implementation of quantum logic gates with trapped ions is affected by various sources of imperfections, which are not captured by the model Hamiltonian (1) [22]. However, even within this model, the unitary $U$, Eq. (3), deviates in general from the target gate unitary $U_{0}$, Eq. (7). We distinguish three types of such intrinsic gate imperfections: Residual qubit-phonon entanglement, over- and under-rotation errors, and crosstalk. To formalize this distinction, we factorize the gate unitary as $U=U_{\alpha} U_{\chi}$, where

$$
U_{\alpha}=e^{\sum_{i=1}^{N} \phi_{i} \sigma_{i}^{x}}, \quad U_{\chi}=e^{i \sum_{i<i^{\prime}=1}^{N} \chi_{i, i^{\prime}} \sigma_{i}^{x} \sigma_{i^{\prime}}^{x}} .
$$

Deviations of $U_{\alpha}$ from the identity, $U_{\alpha} \neq 1$, imply that qubits and phonons are entangled at the end of the gate operation. Over- and under-rotation errors as well as crosstalk occur for $U_{\chi} \neq U_{0}$.

The adverse effect of residual qubit-phonon entanglement can be quantified in terms of the average fidelity [58] per gate, which we define as

$F=\left[\int d \Psi\left\langle\Psi\left|U_{\chi}^{\dagger} \operatorname{tr}_{\mathrm{ph}}\left(U|\Psi\rangle\langle\Psi| \otimes \rho_{\mathrm{th}} U^{\dagger}\right) U_{\chi}\right| \Psi\right\rangle\right]^{1 / G}$,

where the trace is over the motional degrees of freedom, $\rho_{\text {th }}$ denotes a thermal state of phonons, and the integration is over the Fubini-Study measure [59]. We note that usually the fidelity is defined with respect to the ideal target gate, i.e., with $U_{x}$ replaced by $U_{0}$, to explicitly include overand under-rotation errors as well as crosstalk. In contrast, we quantify these types of errors separately as detailed below. To account for the exponential dependence of the total fidelity $F_{\text {tot }}$ on the number $G$ of gates, which are being performed in parallel — which in the cases of interest to us scales with the number of qubits $G \sim N$-we include an exponent $1 / G$ in the definition of the fidelity per gate.

The expression for the average fidelity per gate in Eq. (10) can be made more explicit in two steps: the first step is to perform the trace over phonon modes of the thermal density matrix $\rho_{\text {th }}$, multiplied with qubit-statedependent displacement operators, which are contained in $U$ and $U^{\dagger}$ and correspond to the first term in the exponent in Eq. (3) [22]. Second, the integral over random initial qubit states $|\Psi\rangle$ can be evaluated explicitly by replacing it by a sum over a discrete basis of unitary operators [58].
This procedure yields an expression for the infidelity per gate $\delta F=1-F$, which, in the limit of high fidelity, takes the form

$$
\delta F=\frac{4}{5 G} \sum_{i, n=1}^{N}\left|\alpha_{i}^{n}\right|^{2}\left[2 n_{\mathrm{th}}\left(\omega_{n}\right)+1\right],
$$

where $\alpha_{i}^{n}$ is the qubit-phonon coupling defined in Eq. (5), and $n_{\mathrm{th}}\left(\omega_{n}\right)=1 /\left(e^{\hbar \omega_{n} / T}-1\right)$ is the average thermal occupation of phonons in the mode with frequency $\omega_{n}$ at temperature $T$. For simplicity, we assume in the following that $n_{\text {th }}\left(\omega_{n}\right)=0.5$ for all phonon modes.

Over- and under-rotation errors of the gate $U$ correspond to deviations of the qubit-qubit coupling $\chi_{i, i^{\prime}}$ from the desired value $\chi_{i, i^{\prime}}^{0}$ for pairs of qubits $\left(i, i^{\prime}\right)$, which are contained in $I$ in Eq. (7), i.e., which are affected by the ideal gate $U_{0}$. In contrast, crosstalk is due to unwanted nonzero qubit-qubit couplings for pairs of qubits that are not contained in $I$. To separate these types of errors, we factorize the qubit-qubit coupling in Eq. (3) as $U_{\chi}=U_{1} U_{C}$ where

$$
U_{1}=\prod_{\left(i, i^{\prime}\right) \in I} e^{i \chi_{i, i^{\prime}} \sigma_{i}^{x} \sigma_{i^{\prime}}^{x}}, \quad U_{C}=\prod_{\left(i, i^{\prime}\right) \in I^{\prime}} e^{i \chi_{i, i^{\prime}} \sigma_{i}^{x} \sigma_{i^{\prime}}^{x}},
$$

and where $I^{\prime}$ contains all ordered pairs of qubits that are not included in $I$. We quantify these gate errors in terms of the diamond norm of the error superoperators $\mathcal{E}_{1}=\mathcal{U}_{1}-\mathcal{U}_{0}$ and $\mathcal{E}_{C}=\mathcal{U}_{C}-\mathcal{I}$, where $\mathcal{U}_{b}(\rho)=U_{b} \rho U_{b}^{\dagger}$ for $b=0,1, C$, and $\mathcal{I}(\rho)=\rho[23,60,61]$. Bounds on the errors per gate are given by [62]

$$
\begin{gathered}
\frac{1}{G}\left\|\mathcal{E}_{1}\right\|_{\diamond} \leq \delta \chi=\frac{2}{G} \sum_{\left(i, i^{\prime}\right) \in I}\left|\chi_{i, i^{\prime}}-\chi_{i, i^{\prime}}^{0}\right| \\
\frac{1}{G}\left\|\mathcal{E}_{C}\right\|_{\diamond} \leq C=\frac{2}{G} \sum_{\left(i, i^{\prime}\right) \in I^{\prime}}\left|\chi_{i, i^{\prime}}\right|,
\end{gathered}
$$

where we defined the over- and under-rotation error $\delta \chi$ and the crosstalk $C$.

\section{OPTICAL DESIGN OF LOCALIZED PHONON MODES}

Our goal is to design localized phonon modes in long laser-cooled ion strings, which, as we go on to show in Secs. IV and V, enable the implementation of scalable and parallel entangling quantum gates. In particular, we engineer specific types of mode matrices $M_{\alpha, i}^{n}$ by using optical tweezers that are focused on the equilibrium positions of specific ions and thus pin these ions as we describe in Appendix A. The tweezers are realized by Gaussian laser 
beams along the $y$ direction as illustrated in Fig. 1(a). In the vicinity of the focuses of the tweezers, the optical potential can be approximated as harmonic. The optical trapping frequency along the beam axis is negligible. In contrast, the optical trapping frequency $\omega_{0, i}$ along the transverse $x$ and the longitudinal $z$ directions is determined by the beam intensity and waist at the position of ion $i$, and we assume that $\omega_{0, i}$ does not depend on the internal state of the ions and can take on values up to $\omega_{0, i} \lesssim 0.4 \omega_{x}$ for typical transverse trapping frequencies $\omega_{x}=2 \pi \times 3 \mathrm{MHz}$. In Appendix B, we discuss experimental requirements to realize such strong qubit-state-independent optical potentials for different ionic species.

In the following, we first consider localized phonon modes of a single pair of pinned, neighboring ions in a long chain. This allows us to delimit the regime of strong pinning in which the phonon modes of the pinned ions decouple from the modes of the spectator ions. We then illustrate these ideas with concrete examples of finite and infinite chains.

\section{A. Pinning a single ion pair in a long chain}

To engineer transverse $x$ phonon modes that are localized on a pair of neighboring ions $i$ and $i+1$ and which can thus be used to perform an entangling gate on this pair, we consider a situation in which the pinning on the ions forming the pair is the same, $\omega_{0, i}=\omega_{0, i+1}$, whereas the remaining spectator ions are not pinned. The residual Coulomb interaction (see Appendix A for details) has two effects: First, the interaction between the pinned ions leads to the formation of localized COM and stretch modes given by

$$
\begin{aligned}
M_{x, i^{\prime}}^{\mathrm{COM}} & \approx\left(\delta_{i^{\prime}, i}+\delta_{i^{\prime}, i+1}\right) / \sqrt{2}, \\
M_{x, i^{\prime}}^{\text {stretch }} & \approx\left(\delta_{i^{\prime}, i}-\delta_{i^{\prime}, i+1}\right) / \sqrt{2} .
\end{aligned}
$$

These are the desired modes to implement two-qubit entangling gates. The frequency splitting of these modes is determined by the Coulomb interaction, $\omega_{\mathrm{COM}}-\omega_{\text {stretch }} \approx$ $e^{2} /\left(4 \pi \epsilon_{0} d_{i, i+1}^{3} m \omega_{x}\right)$, where $d_{i, i^{\prime}}=\left|z_{i, 0}-z_{i^{\prime}, 0}\right|$ is the distance between the equilibrium positions $z_{i, 0}$ of the ions along the trap $z$ axis, $e$ is the elementary charge and $\epsilon_{0}$ is the vacuum permittivity. Second, the interaction between pinned and spectator ions slightly admixes oscillations of the spectator ions to the localized modes, i.e., the mode vectors Eq. (15) acquire nonzero amplitudes on ions $i^{\prime} \notin$ $\{i, i+1\}$. This unwanted effect is strongly suppressed if the difference between the squares of the local oscillation frequencies of the pinned and spectator ions, as given in Eq. (A3), is large in comparison to their residual Coulomb interaction.

More generally, for transverse $x$ phonon modes of a chain of ions with mean spacing $d$, the regime of strong pinning in which localized phonon modes emerge can be conveniently characterized in terms of two dimensionless parameters:

$$
\epsilon=\sqrt{\frac{e^{2}}{4 \pi \epsilon_{0} d^{3} m \omega_{x}^{2}}}, \quad v_{0}=\frac{\omega_{0}}{\omega_{x}},
$$

where for simplicity we assume that all ions are pinned with the same optical trapping frequency $\omega_{0}$. As detailed in Appendix A, localized COM and stretch modes of a pair of pinned ions decouple from the motion of spectator ions if $\nu_{0}^{2} / \epsilon^{2} \gg 1$. While we focus here on pairs of neighboring ions, we note that the same criterion applies for pairs of pinned ions at a larger distance.

In experiments, $\epsilon$ is typically a small parameter. For example, we obtain $\epsilon \approx 0.07$ for a spacing of $d=10 \mu \mathrm{m}$ in a chain of ${ }^{24} \mathrm{Mg}^{+}$ions with a transverse trapping frequency of $\omega_{x}=2 \pi \times 5.5 \mathrm{MHz}$, or ${ }^{40} \mathrm{Ca}^{+}$ions with $\omega_{x}=2 \pi \times 4.2 \mathrm{MHz}$. The ratio $v_{0}^{2} / \epsilon^{2}$ can be increased by either increasing the tweezer trapping frequency $\omega_{0}$ or by increasing the spacing of the ions $d$.

\section{B. Localized phonon modes in a finite chain}

As an example, we consider a chain of $N=60$ ions as depicted in Fig. 2(a). We assume harmonic confinement along the $\operatorname{trap} z$ axis with trapping frequency $\omega_{z}$. Starting from the sixth ion, optical tweezers are arranged to divide the chain into groups of $p=6$ ions. The resulting mode matrix $M_{x, i}^{n}$ for $i, n \in\{1, \ldots, N\}$ for oscillations in the transverse $x$ direction is shown in Fig. 2(b). Orange boxes mark pinned ions, and gray boxes indicate buffer ions at the ends of the chain. The spacing of these ions deviates strongly from the approximately uniform spacing in the center of the chain, and we exclude them from gate operations. As explained above, the residual Coulomb coupling leads to the formation of localized COM and stretch modes of the pinned pairs. In the figure, COM and stretch modes are distinguished by the same color of $M_{x, i}^{n}$ and $M_{x, i+1}^{n}$ for two neighboring ions that oscillate in phase and different colors for oscillations with opposite phase. As an additional effect, which is due to the long-range character of the residual Coulomb interaction, the localized modes of distinct pinned pairs hybridize, where the number of ions in pinned pairs, which is 18 for this example, determines the number of hybridized modes. These modes are highlighted by blue shading in the figure.

To perform entangling gates on pairs of pinned ions, the hybridization of localized phonon modes is in principle not desirable. However, as long as the frequency splitting of the localized modes due to the hybridization is so small that it cannot be resolved on the time scale of the gate operation, the hybridization has only a small effect on the gate performance. This picture generalizes the concept of local oscillation modes from single ions [63] to pairs of ions. 

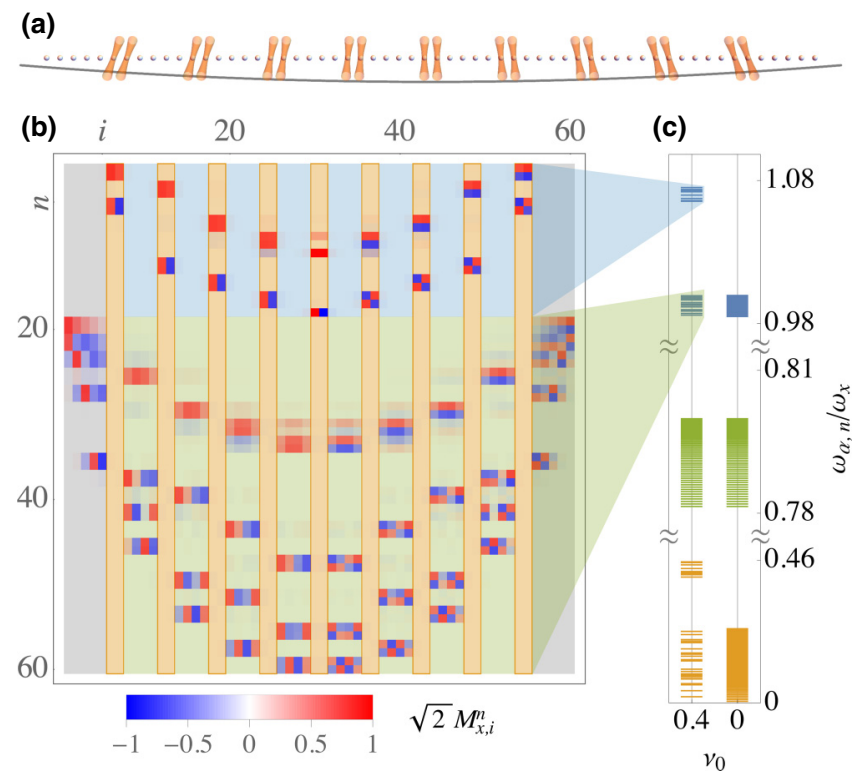

FIG. 2. (a) $N=60$ ions in an array of optical tweezers, which subdivides the chain into groups of $p=6$ six ions. The first and last five buffer ions are not used as qubits. (b) Mode matrix $M_{x, i}^{n}$ for transverse $x$ modes. Orange and gray shading indicates pinned and buffer ions, respectively. Blue shading marks modes that are superpositions of localized COM and stretch modes of pairs of pinned ions. (c) Phonon-mode spectrum for $v_{0}=0.4$ and, for comparison, without tweezers, i.e., for $v_{0}=0$. Transverse $x$ and $y$ modes and longitudinal $z$ modes are shown, respectively, in blue, green, and orange. We assume that the ratio of transverse trapping frequencies is given by $\omega_{y} / \omega_{x}=0.8$.

The order of mode indices $n$ in Fig. 2(b) reflects the mode frequency, with $n=1$ corresponding to the mode with the highest frequency. For transverse oscillations, this is a COM-like mode, which is given here by the in-phase oscillation of local COM modes of the outermost pairs. The second-highest frequency mode with $n=2$, in turn, corresponds to a superposition of the local COM modes of the outermost pairs with opposite phase. For this example, due to the reflection symmetry with regard to the center of the trap, these hybridized modes are equal superpositions of oscillations of pairs to the left and right of the trap center. Superpositions of local COM modes are followed at lower frequencies by superpositions of local stretch modes.

The phonon-mode spectrum of the chain, both with and without tweezers, is shown in Fig. 2(c). The spectra of oscillations in the longitudinal $z$ and transverse $x$ direction, which are shown in orange and blue, respectively, are strongly modified in the presence of tweezers with strength $v_{0}=\omega_{0} / \omega_{x}=0.4$ : the spectrum of oscillations along the trap $z$ axis is gapped [34], and the almost dense set of frequencies splits up into several subsets. Most prominently, both for the $z$ and $x$ modes, a subset of modes, which correspond to hybridized COM and stretch modes of the pinned pairs, appear shifted above the remaining mode frequencies. For the transverse $x$ direction, the assignment between mode frequencies and mode vectors is indicated with blue and green shading. The spectrum of transverse $y$ modes, which is shown in green in Fig. 2(c), is not affected by the tweezers because we neglect the trapping along the direction of the tweezer beam.

\section{Phonon band structure for infinite chains}

To demonstrate the inherent scalability of our approach, we also present theoretical results for tweezer gates in infinite ion chains as illustrated in Fig. 3(a). In the simultaneous limit $N \rightarrow \infty$ and $\omega_{z} \rightarrow 0$, the system acquires discrete translational invariance under the transformation $i \mapsto i+p$, where $p$ is the size of the unit cell of the spatially periodic arrangement of tweezers. We note that discrete translational symmetry is also realized in ring traps [64]. To account for this translational invariance, it is convenient to label the positions of ions as $(l, i)$ with unit-cell index $l \in \mathbb{Z}$ and position $i \in\{1, \ldots, p\}$ within a unit cell. The ions at positions $i \in\{1,2\}$ are pinned whereas the remaining ions at $i \in\{3, \ldots, p\}$ are not pinned. (a)

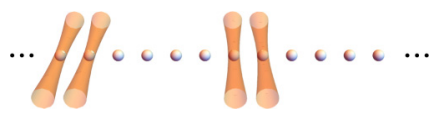

(b)
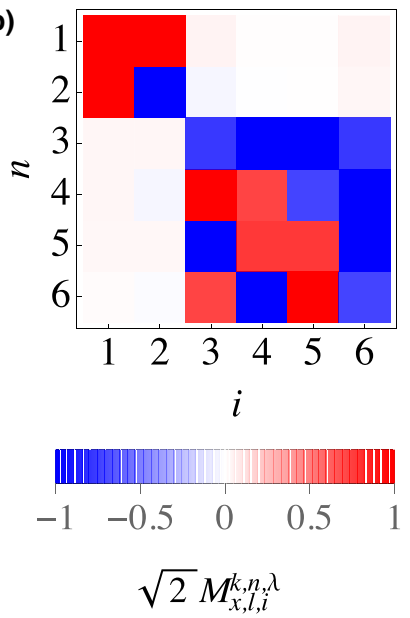

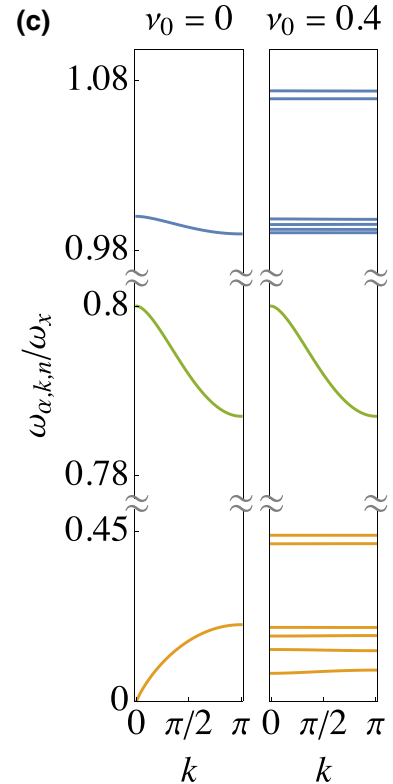

FIG. 3. (a) Infinite ion chain with optical tweezers forming a periodic array with unit cell size $p=6$. (b) Mode matrix $M_{x, l, i}^{k, n}$ for $l=k=0$ and $\lambda=1$. COM and stretch modes of the pinned ions are clearly decoupled from the ions that are not pinned. (c) Phonon-mode spectra for an infinite chain without and with optical tweezers, for $v_{0}=0$ and $v_{0}=0.4$, respectively. Transverse $x$ and $y$ modes and longitudinal $z$ modes are shown, respectively, in blue, green, and orange. The spatial periodicity imposed by tweezers causes the dense bands of the $x$ and $z$ modes in the infinite system to split up into $p=6$ bands, where the highest two bands correspond to COM and stretch modes of pinned pairs. 
The normal modes of a periodic ion crystal can be found by using concepts from band theory of electrons in solids as detailed in Appendix A 2. In particular, translational invariance with respect to the unit-cell index $l$ can be accounted for by representing the phonon modes as plane waves proportional to $e^{i k l}$ with quasimomentum $k$. Because the phonon-mode vectors are real, the quasimomentum can be restricted to the interval $k \in[0, \pi]$. We note that the plane-wave representation implies that phonon modes are stretched out over the entire chain. In other words, the localized COM and stretch modes of pinned pairs in different unit cells hybridize uniformly.

The phonon-mode structure for infinite systems is illustrated in terms of the mode matrix $M_{\alpha, l, i}^{k, n, \lambda}$ (see Appendix A 2) in Fig. 3(b) for $\alpha=x, l=0, k=0$, and $\lambda=1$. Within a unit cell, there is a clear separation between modes $n=1$ and $n=2$, which correspond, respectively, to COM and stretch oscillations of the pinned ions, and the modes with $n \in\{3, \ldots, p\}$, which involve the ions that are not pinned.

As shown in Fig. 3(c), modifications of the mode frequencies due to tweezers are particularly clear in infinite systems: The bands, which are formed by $z$ and $x$ modes, split up into $p=6$ bands in the presence of tweezers. In particular, COM and stretch modes of the pinned pairs hybridize between unit cells to form bands. The widths of these bands are vanishingly small on the scale of the figure. Moreover, close inspection reveals that the width of the stretch band is smaller than the width of the COM band by one order of magnitude. These features of the COM and stretch bands can be understood in terms of perturbation theory in the small parameter $\epsilon^{2} / v_{0}^{2}$ as we show in Appendix A 2. The perturbative treatment shows that the widths of the COM and stretch bands are proportional to $\epsilon^{2} \omega_{x} / p^{3}$ and proportional to $\epsilon^{2} \omega_{x} / p^{5}$, respectively. That is, the widths are suppressed with the size of the unit cell $p$, with an even stronger suppression for the stretch band.

\section{ENTANGLING TWEEZER GATES}

The optical design of phonon modes as described above forms the basis to implement parallel two-qubit entangling gates. In the following, we discuss the requirements for and performance of tweezer gates in infinite as well as finite ion chains.

\section{A. Infinite chains}

We consider a periodic array of pinned pairs of ions, which are separated by $p-2$ spectator ions, and we aim at performing two-qubit entangling gates on all pairs of pinned ions in parallel. As illustrated in Fig. 3(b), the spectrum of transverse $x$ phonon modes comprises $p$ bands with mean frequencies $\omega_{n}$ and bandwidths $\Delta_{n}$.

We aim at implementing gates using dominantly the $\mathrm{COM}$ or stretch bands with mean frequencies $\omega_{1}=\omega_{\mathrm{COM}}$ and $\omega_{2}=\omega_{\text {stretch }}$, respectively. This can be achieved if the resolved-sideband condition

$$
\left|\mu-\omega_{n}\right| \ll \mu, \omega_{n},
$$

is met where $\mu>\omega_{1}$ for the COM band with $n=1$, and $\mu<\omega_{2}$ for the stretch band with $n=2$. The choice of tuning above the COM or below the stretch band ensures that the detuning $\mu$ is as far away as possible from the respective other band, which should not be excited.

Note that contributions from higher bands with $n \in$ $\{3, \ldots, p\}$ to the qubit-phonon coupling [Eq. (5)] and qubit-qubit coupling [Eq. (6)] are strongly suppressed: first, these bands are far detuned with $\mu-\omega_{n} \gtrsim \omega_{0}$ with the frequency of the optical potential $\omega_{0}$; second, the contribution of these bands to the couplings [Eqs. (5) and (6)] is proportional to $\left|\eta_{l, i}^{k, n, \lambda} \Omega_{i}\right| / \omega_{0}$, which is suppressed in the limit of strong pinning by a factor of $\eta_{l, i}^{k, n, \lambda} \sim \epsilon^{2} / \nu_{0}^{2}$ for pinned ions at positions $(l, i)$ as follows from the perturbative treatment presented in Appendix A 2. Moreover, as detailed below we find that typically $\Omega_{i}$ is smaller than $\omega_{0}$ by an order of magnitude. We note that due to the proportionality to $\Omega_{i}$, by setting $\Omega_{i}=0$ for the ions that are not pinned, these do not contribute to the infidelity and the crosstalk.

The precise values of $\mu$ and, in particular, $\tau$, follow from the condition of minimal infidelity. For an isolated pair of ions with COM and stretch-mode frequencies $\omega_{1}$ and $\omega_{2}$, respectively, this can be achieved for $[18,52]$

$$
\left(\mu-\omega_{n}\right) \tau=2 \pi l_{n},
$$

where $l_{n}$ is an integer. Particular choices for gates that use dominantly the COM and stretch modes are given by, respectively, $l_{1}=1$ and $l_{2}=2$, and $l_{1}=-2$ and $l_{2}=-1$. The resulting detunings are

$$
\mu_{\mathrm{COM}}=2 \omega_{1}-\omega_{2}, \quad \mu_{\text {stretch }}=2 \omega_{2}-\omega_{1},
$$

and in both cases the gate duration $\tau=2 \pi /\left(\omega_{1}-\omega_{2}\right)$ is set by the mode splitting. These choices of detunings and gate duration ensure that the displacement of the phonon modes due to the qubit-phonon coupling [Eq. (5)], when considered as a function of the upper limit of integration $\tau^{\prime} \in[0, \tau]$, performs a closed loop in phase space [49-52].

In the present case of an infinite chain, Eq. (18) remains valid in the case of two narrow bands, if the bandwidths $\Delta_{n}$ are sufficiently small in the sense that $\Delta_{n} \tau \ll 1$. However, clearly this condition cannot be met for all frequencies $\omega_{k, n}$ which form a band and correspond to different quasimomenta $k$. For the results we show below, we first fix $\tau$ according to Eq. (18) for either the COM or the stretch band by setting either $l_{1}=1$ or $l_{2}=-1$ and by choosing $\omega_{n}$ as the mean frequency of the respective band, and we then determine numerically the value of $\mu$ that yields the lowest infidelity, which typically deviates only slightly 
from the values given in Eq. (19). The Rabi frequency $\Omega_{l, i}=\Omega_{0}$ on the pinned ions is chosen according to the condition $\chi_{i, i^{\prime}}^{l, l^{\prime}}= \pm \pi / 4$ if $(l, i)$ and $\left(l^{\prime}, i^{\prime}\right)$ are neighboring pinned ions, i.e., $l^{\prime}=l$ and $i^{\prime}=i+1$. For the remaining ions, which are not pinned, we set $\Omega_{l, i}=0$.

We first consider the implementation of parallel gates that use the stretch band in an ion chain with $p=6$, $\epsilon=0.07$, and $\nu_{0}=0.4$. To minimize the infidelity we choose $\mu / \omega_{x} \approx 1.065$ and $\omega_{x} \tau \approx 1.37 \times 10^{3}$ as described above. The required Rabi frequency to perform a maximally entangling gate is given by $\eta_{0} \Omega_{0} / \omega_{x} \approx 4.75 \times 10^{-3}$, where the dimensionless factor

$$
\eta_{0}=k_{L, x} \sqrt{\hbar /\left(2 m \omega_{x}\right)}
$$

contains all parameters in the definition of the Lamb-Dicke parameter matrix, Eq. (2), which are specific to different ionic species.

In Fig. 4(a), we show the qubit-phonon coupling, Eq. (5), as a function of the upper limit of integration $\tau^{\prime} \in[0, \tau]$ and for $l=i=1, n=1,2$, and $k \approx 1.55$, which corresponds to the mean frequency $\omega_{2}$ of the stretch band. According to Eq. (3), the actual displacement depends on the state of the qubit at position $(l, i)$, and is opposite for the states $| \pm\rangle_{i}$ with $\sigma_{i}^{x}| \pm\rangle_{i}= \pm| \pm\rangle_{i}$. The values of $n$ and $k$ shown in Fig. 4(a) yield the largest values of the qubit-phonon coupling, i.e., the corresponding modes contribute most to the gate. On the scale of the figure, the qubit-phonon coupling for bands with $n \geq 3$ is not visible. The physical picture described below Eq. (19) is clearly reflected in the figure: the phase-space trajectories for both the stretch and the COM modes shown in the figure are closed, where the trajectory of the COM mode is traversed twice. However, closer inspection of the vicinity of the origin reveals that the trajectories of modes that belong to the $\mathrm{COM}$ band and have different values of the quasimomentum $k$, do not close perfectly. In Fig. 4(b) we show the qubit-phonon coupling at the end of the gate operation as a function of $k$ for both the stretch and the COM bands. According to Eq. (11), the area under these curves determines the infidelity, and the figure shows that the dominant contribution to the infidelity is indeed due to the COM modes. We find $\delta F=5.7 \times 10^{-4}$.

The infidelity is higher for gates that use predominantly the COM band. This is because the width of the COM band is much larger than the width of the stretch band and, therefore, the phase-space trajectories for different $k$ deviate more strongly from perfect closure. In particular, for $\mu / \omega_{x} \approx 1.079$ slightly below the COM band, $\omega_{x} \tau \approx$ $1.37 \times 10^{3}$, and $\eta_{0} \Omega_{0} / \omega_{x} \approx 4.77 \times 10^{-3}$, we obtain $\delta F=$ $2.1 \times 10^{-3}$, which is higher than the value we obtain for the stretch band by an order of magnitude.

We next analyze over- and under-rotation errors and crosstalk as defined in Eqs. (13) and (14), respectively. For the implementation of gates we discuss in this section, the
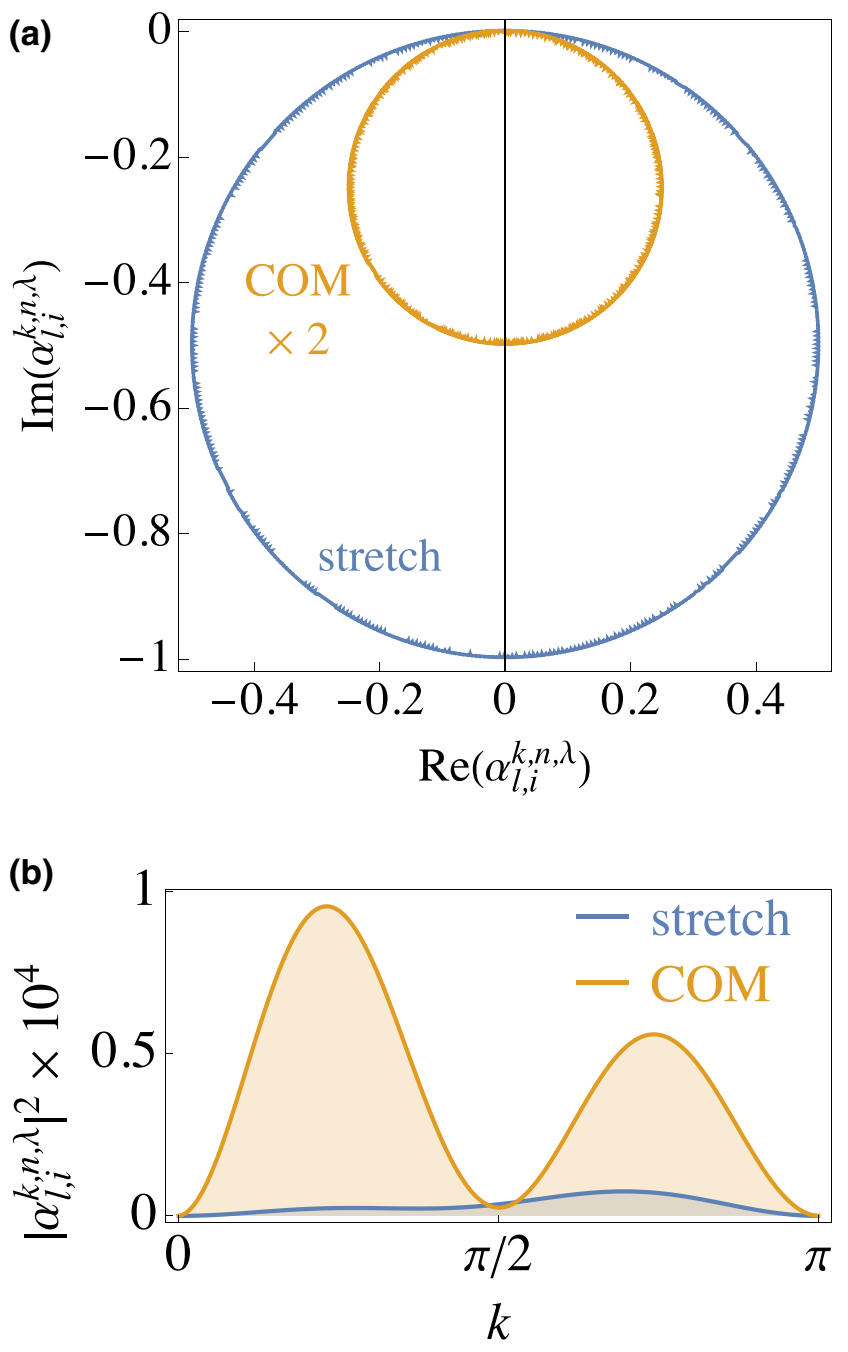

FIG. 4. Phase-space trajectories for parallel entangling gates in an infinite chain. (a) Contribution to the displacement of the phonon mode $k, n, \lambda$ due to its coupling, Eq. (5), to the qubit at position $l=i=1$, which is assumed to be in the +1 eigenstate of $\sigma_{l, i}^{x}$. For the chosen detuning close to $\mu_{\text {stretch }}$ in Eq. (19), the stretch mode with $n=\lambda=2$ and $k \approx 1.55$ such that $\omega_{k, 2}=\omega_{2}$ equals the mean frequency of the stretch band experiences the strongest displacement. The phase-space trajectory of the COM mode with the same values of $k$ and $\lambda$ forms a smaller circle, which is traversed twice. Trajectories of modes with $n \geq 3$ are not visible on the scale of the figure. (b) Closer inspection reveals that the phase-space curves for different values of $k$ are not perfectly closed. The area under the squared modulus of the qubit-phonon coupling at the end of the gate operation, shown here for $n=\lambda=2$, determines the infidelity (11). Parameters of the ion chain are $p=6, v_{0}=0.4$, and $\epsilon=0.07$.

Rabi frequency is chosen such that $\chi_{i, i^{\prime}}=\chi_{i, i^{\prime}}^{0}$ for $\left(i, i^{\prime}\right) \in$ $I$, i.e., the over- and under-rotation error vanishes exactly, $\delta \chi=0$.

However, there is unwanted crosstalk corresponding to nonzero qubit-qubit couplings between ions that belong to distinct pairs. The dominant contributions to crosstalk are 
due to (i) unwanted excitation of the stretch or COM band, and (ii) the finite width of these bands. Concerning (i), we note that for a given detuning, say $\mu>\omega_{1}$, the COM and stretch bands induce ferromagnetic and antiferromagnetic couplings. In other words, they yield contributions to $\chi_{i, i^{\prime}}^{l, l^{\prime}}$ with opposite sign, which thus partially cancel each other. This partial cancellation has to be compensated by increasing the Rabi frequency $\Omega_{0}$ to achieve $\chi_{i, i^{\prime}}^{l, l^{\prime}}= \pm \pi / 4$ on the target ions, which then, however, also increases unwanted couplings, which contribute to the crosstalk, Eq. (14). This effect is suppressed by tuning close to a sideband Eq. (17), i.e., by dominantly exciting either the COM or the stretch band.

With regard to (ii), the fact that a finite bandwidth leads to crosstalk can be understood intuitively from the fact that the effective coupling between local (within single unit cells) COM or stretch modes determines the bandwidth. Crosstalk is negligible if the effective coupling and thus the bandwidth is much smaller than all other relevant scales. In particular, the finite bandwidth can be neglected and $\omega_{k, n}$ for the COM and stretch bands can be replaced by the respective central frequencies $\omega_{n}$ in Eq. (6) if

$$
\left|\mu-\omega_{n}\right| \gg \Delta_{n} .
$$

We note that by Eq. (17) this also implies that $\mu, \omega_{n} \gg$ $\Delta_{n}$. If we combine this condition with Eq. (18) for the gate duration, we find the intuitive criterion that to minimize crosstalk the gate should be performed fast on the timescale that is set by the effective coupling between pinned pairs. This picture generalizes the concept of local oscillation modes from single ions [63] to pairs of ions. We note that the mentioned condition can always be met efficiently by increasing the size of the unit cell $p$.

For the gate shown in Fig. 4, we find $C=4.1 \times 10^{-2}$. The crosstalk for a gate using the COM band and with $p=6$ is $C=1.7 \times 10^{-1}$, i.e., again one order of magnitude higher than for the stretch band. The crosstalk can be reduced by increasing the unit-cell size $p$. In particular, for gates that use the stretch band and for $p \geq 9$, we obtain $C<10^{-2}$ such that $C / \chi_{i, i^{\prime}}<1 \%$ for $\left(i, i^{\prime}\right) \in I$.

Finally, we note that while the long-range character of the residual Coulomb interaction has the adverse effect of leading to crosstalk between distant qubits, it can also be utilized to implement gates between qubits that are not nearest neighbors. This can be achieved, for example, by pinning the ions at positions 1 and $q$, where $2<q \ll p$ within each unit cell. This leads to a reduction of the splitting between the COM and stretch bands by a factor proportional to $1 /(q-1)^{3}$, and the gate duration increases correspondingly according to Eq. (18).

\section{B. Gate performance}

We study how infidelity, crosstalk, and gate duration depend on the tweezer pinning strength as measured by the dimensionless optical trapping frequency $v_{0}$, and the strength of the residual Coulomb coupling $\epsilon$ in Fig. 5. The main panel of this figure shows the infidelity $\delta F$ for $p=6$ and for optimized choices of gate duration and detuning as discussed above. For large values of $\epsilon$ in the white region, the gap that separates the COM and stretch bands is more than half of the gap that separates the stretch band from the bands of the ions that are not pinned and, therefore, the pinned ions are not sufficiently decoupled from the ions that are not pinned. In this region, gates cannot be performed with reasonable infidelity. High-fidelity gates can be performed for values of $\epsilon$ below the black diagonal line, which corresponds to a threshold value of $\delta F=10^{-3}$.

We next analyze how the gate speed and crosstalk are affected by the optical trapping frequency $v_{0}$ for a fixed value of the infidelity $\delta F=10^{-3}$, i.e., for $\epsilon$ on the black diagonal line in Fig. 5. The inset in the upper left corner of the figure shows the gate duration for various values of the unit-cell size $p$. The gate duration is set by the splitting between COM and stretch bands, which according to Eq. (A16) is proportional to $\epsilon^{2}$ for $\epsilon \ll 1$. The resulting analytical prediction of the scaling $\tau \sim 1 / \epsilon^{2} \sim 1 / v_{0}^{2}$ on the line $\delta F=10^{-3}$ is shown as a dashed gray line in the inset and agrees well with the numerical data. (The vertical offset between the gray line and the numerical data is introduced to improve the visibility.) For $v_{0}$ in the range from 0.1 to 0.4 , we obtain gate durations between $\omega_{x} \tau=0.05 \times 10^{4}$ and $10^{4}$. For a typical value of $\omega_{x}=$

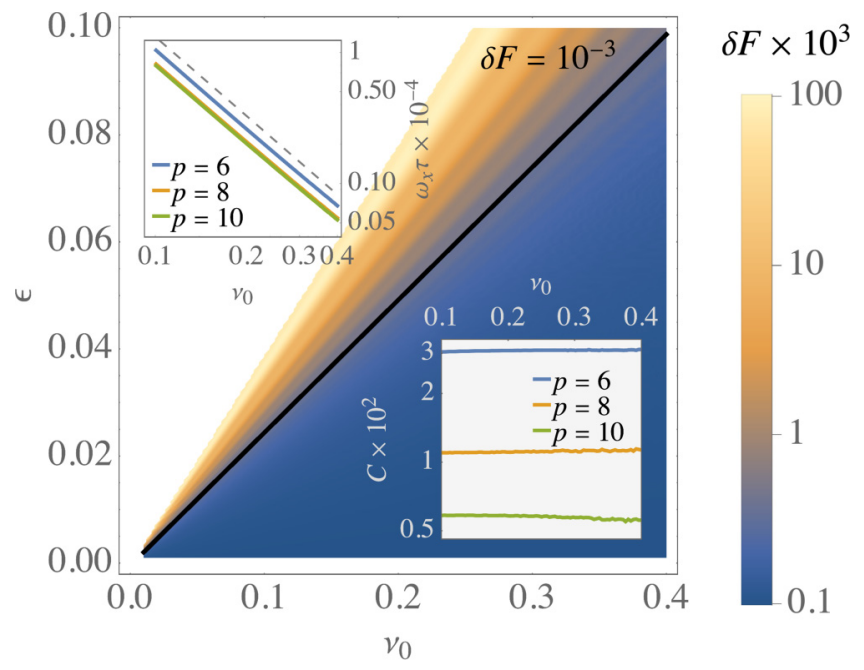

FIG. 5. Gate performance for an infinite chain. The main panel shows the dependence of the infidelity on the parameters $v_{0}$ and $\epsilon . \delta F=10^{-3}$ on the black line across the diagonal. In the white region, the optical potential is insufficient to decouple the pinned ions from the other ions in the chain. The insets show the gate duration $\tau$ and the crosstalk $C$ for fixed infidelity $\delta F=10^{-3}$. 
TABLE I. Tweezer parameters and scattering-induced infidelity for ground-state qubit encoding in different ionic species. The parameters $\epsilon=0.07, v_{0}=0.4$, and $\omega_{x}=2 \pi \times 3 \mathrm{MHz}$ are fixed for all species, and the numerical aperture is assumed to be 0.7 (see Appendix B for details). The tweezer wavelength is chosen to result in a close-to-optimal scattering infidelity while being experimentally accessible in terms of both power per tweezer spot in the second column and available laser sources.

\begin{tabular}{lcccc}
\hline \hline & $\begin{array}{c}\text { Wavelength } \\
(\mathrm{nm})\end{array}$ & $\begin{array}{c}\text { Optical } \\
\text { power } \\
(\mathrm{mW})\end{array}$ & $\begin{array}{c}\text { Scattering } \\
\text { infidelity } \\
\times 10^{3}\end{array}$ & $\begin{array}{c}\text { Interion } \\
\text { distance } \\
(\mu \mathrm{m})\end{array}$ \\
\hline${ }^{24} \mathrm{Mg}^{+}$ & 400 & 6.4 & 4.9 & 15 \\
${ }^{40} \mathrm{Ca}^{+}$ & 532 & 14.5 & 12.0 & 12.6 \\
${ }^{88} \mathrm{Sr}^{+}$ & 580 & 40.2 & 30.2 & 9.7 \\
${ }^{171} \mathrm{Yb}^{+}$ & 532 & 202.2 & 38.2 & 7.8 \\
${ }^{138} \mathrm{Ba}^{+}$ & 675 & 90.0 & 55.0 & 8.3 \\
\hline \hline
\end{tabular}

$2 \pi \times 3 \mathrm{MHz}$, this corresponds to gate durations ranging from 27 to $531 \mu \mathrm{s}$.

The crosstalk for fixed infidelity $\delta F=10^{-3}$ is shown in the inset in the lower right corner in Fig. 5. While the crosstalk remains approximately constant as a function of $v_{0}$, it can be suppressed by increasing the unit-cell size $p$. As already stated above, we find $C<10^{-2}$ for $p \geq 9$.

In an experimental implementation, an important contribution to the infidelity is due to spontaneous scattering of photons of the tweezer beams. As discussed in Appendix $\mathrm{B}$, promising candidates to implement tweezer gates are ${ }^{24} \mathrm{Mg}^{+}$ions, for which the infidelity due to scattering of photons is on the order of $10^{-3}$ for reasonable dimensionless parameters $\epsilon$ and $v_{0}$. Tweezer parameters and scattering infidelities for several ionic species are summarized in Table I. The infidelity due to scattering can be decreased further by decreasing the tweezer intensity and thus the dimensionless optical trapping frequency $v_{0}$. As illustrated in Fig. 5, this results in an increase of the gate infidelity, which, however, can be compensated by employing optimal coherent control as described in Appendix E.

\section{Finite chains}

Here we show how the methods for implementing parallel tweezer gates can be applied in finite 1D ion strings. For concreteness, we assume harmonic trapping along the trap $z$ axis. In contrast to before the Hamiltonian in question is no longer invariant under discrete translations, which leads to stark changes in the mode spectrum as well as the mode functions themselves (see the discussion around Fig. 2). As we point out further below this is not necessarily a negative aspect and indeed further improves the gate performance. For this, the detunings $\mu_{i}$, the Rabi frequencies $\Omega_{i}(t)$ in Eq. (1), and the gate duration have to be controlled for each ion pair. This is due to the fact that for each pair of target ions and in order to fulfill Eq. (18) one has to identify the corresponding COM and stretch mode and choose the detuning and the gate duration accordingly. These deviations are strongest for ions at the ends of the chain where the interion distances are considerably larger than for the rest of the chain. Therefore, we introduce several buffer ions on each end, which are not used for quantum computation. We choose the number of buffer ions such that the relative standard deviation for the interion distances of the other ions lies below $10 \%$. Furthermore, we choose the axial trapping frequency $\omega_{z}$ such that the mean interion distance of the nonbuffer ions corresponds to $\epsilon=0.07$, i.e., the value that we choose for the infinite system.

In Fig. 6 we show numerical results for a system of 130 ions, 15 of which on each end of the chain are used as buffer ions. We choose $p=6$ to implement a total number of 17 maximally entangling gates in parallel, where the leftmost and the rightmost gates are performed on the ions at positions $i=16,17$ and $i=112,113$, respectively. This configuration of gates is not symmetric with respect to the center of the trap. However, this asymmetry does not affect the gate performance: indeed, the performance of the parallel gates for the asymmetric configuration, which we discuss in detail below, is comparable to the gate performance for the symmetric configuration that can be realized by shifting all tweezers by one ion to the right while keeping the total number of gates at 17 .
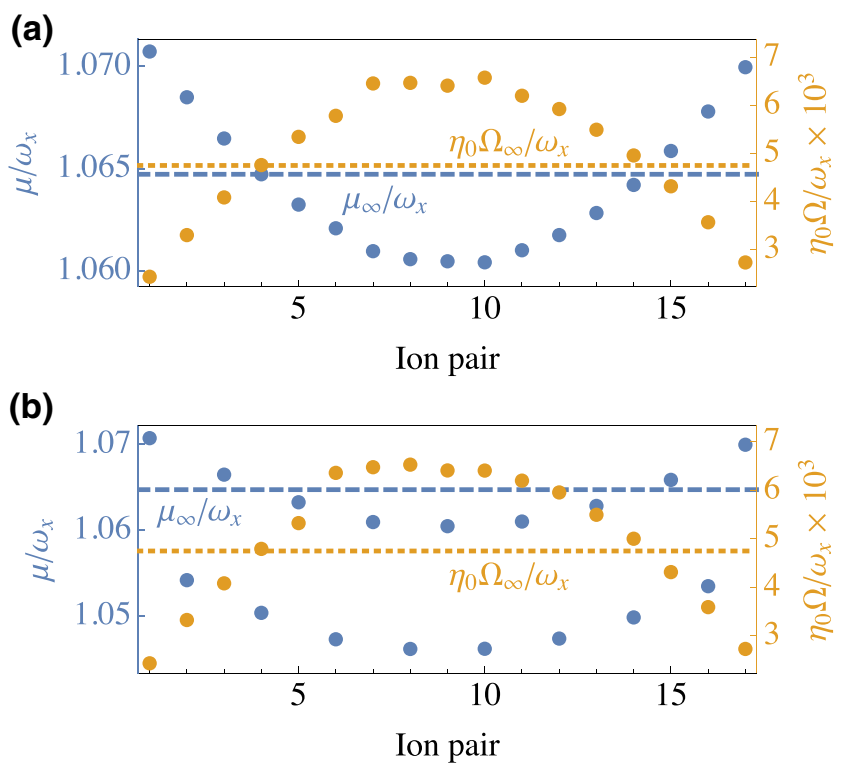

FIG. 6. Tweezer gates in finite chains. We show individual detunings and Rabi frequencies for 17 maximally entangling tweezer gates targeting the stretch mode along a chain of 130 ions with 15 buffer ions on each side $(p=6, \epsilon \approx 0.07)$. Optical tweezers generate a trapping frequency that is uniform $v_{0}=0.4$ and alternating between $v_{01}=0.4$ and $v_{02}=0.36$ for (a) and (b), respectively. For comparison, we also show the detuning $\mu_{\infty}$ and Rabi frequency $\Omega_{\infty}$ for an infinite chain with $\epsilon=0.07$ and $v_{0}=0.4$. 
The two panels in Fig. 6 correspond to different schemes of optical pinning by the tweezers: in (a), each pair of target ions is pinned with the same tweezer strength $v_{0}=0.4$, whereas in (b), tweezer strengths alternate between the values $v_{01}=0.4$ and $v_{02}=0.36$. For both cases we show the detuning and the required Rabi frequency for each gate along the chain. As for the infinite chain, the detunings are optimized with regard to optimal infidelity around the estimate given by Eq. (19) for the stretch mode of each pair. The required detunings lie around the value obtained in the infinite case (blue dashed line in Fig. 6) but are lower in the center and higher at the edge of the chain. This is due to the disparity in the mode frequencies: as indicated in Fig. 2, modes that are localized at the edge of the ion chain have higher frequencies and smaller gaps compared to those at the center. The maximal gate duration $\omega_{x} \tau=2670$ is the same for Figs. 6(a) and 6(b) and occurs at the edge of the ion chain. This can be attributed to the difference in the interion distances that influence the gap between the $\mathrm{COM}$ and the stretch mode of a given ion pair: larger distances implicate weaker interactions and hence a smaller gap leading to slower gates and vice versa [see Eq. (18)]. Furthermore, since the gate duration is larger at the edge, the Rabi frequency, which is also shown in Fig. 6, has to be lower in order to realize a maximally entangling gate.

We note that while the choice of Rabi frequencies shown in Fig. 6 leads to maximally entangling gates with $\chi_{i, i+1}=$ $-\pi / 4$ for all pairs of qubits, different and independent values of $\chi_{i, i+1}$ can be achieved by lowering the individual Rabi frequencies.

As before we quantify the performance of the gate through average infidelity, Eq. (11), and average crosstalk, Eq. (14). For (a) and (b) in Fig. 6 we get $\delta F_{a}=$ $9 \times 10^{-4}, C_{a}=2.8 \times 10^{-2}$ and $\delta F_{b}=1.7 \times 10^{-3}, C_{b}=$ $6.5 \times 10^{-3}$, respectively. If we compare these values with those obtained in the infinite chain, i.e., $\delta F_{\infty}=5.7 \times 10^{-4}$ and $C_{\infty}=4.1 \times 10^{-2}$, we find that while the infidelity is slightly worse, crosstalk is slightly better in the finite case. The former is due to rare outliers with infidelities of order $10^{-2}$ in the middle of the chain whereas the latter stems from the variation in mode frequency (and hence detuning) and the tighter localization of the COM and stretch modes for the different gates. The alternating tweezer frequencies in Fig. 6(b) amplify the latter effect, which leads to further suppression of crosstalk.

\section{Infidelity and over- and under-rotation errors from tweezer misadjustments}

An important question for the experimental implementation of tweezer gates concerns the sensitivity of the gate performance to misadjustments of the optical tweezer array. To address this question, we consider three distinct types of misadjustments: deviations of the focuses of the tweezers from the equilibrium positions of the ions, variations in the optical trapping frequencies due to intensity fluctuations of the tweezers, and misalignment of the tweezers with the $y$ direction. As figures of merit, we study how these imperfections affect the infidelity as well as the over- and under-rotation error.

Including tweezer misadjustments in the optical potential, Eq. (A4), for ion $i \in\{1, \ldots, N\}$ yields

$$
V_{i}^{\mathrm{twz}}\left(\mathbf{r}_{i}\right)=\frac{m}{2}\left(\omega_{0, i}+\delta \omega_{i}\right)^{2}\left|\Pi\left(\theta_{i}, \phi_{i}\right)\left(\delta \mathbf{r}_{i}-\boldsymbol{\delta}_{i}\right)\right|^{2} .
$$

Here, $\delta \omega_{i}$ is a shift of the optical trapping frequency, $\boldsymbol{\delta}_{i}$ is the deviation of the focus of the tweezer from the equilibrium position of the ion $\mathbf{r}_{i, 0}$ in the absence of an optical potential, and $\delta \mathbf{r}_{i}=\mathbf{r}_{i}-\mathbf{r}_{i, 0}$ is the displacement of the ion from $\mathbf{r}_{i, 0}$. In Appendix $C$, we explain how the shift of the equilibrium position of the ion due to deviations of the focus of the tweezer from $\mathbf{r}_{i, 0}$ can be calculated perturbatively. Finally, the angles $\theta_{i}$ and $\phi_{i}$ describe the misalignment of the tweezer beam with the $y$ axis, and $\Pi\left(\theta_{i}, \phi_{i}\right)$ is the projector onto the plane orthogonal to the tweezer beam axis. We assume that fluctuations of the parameters $\delta \omega_{i}, \boldsymbol{\delta}_{i}, \theta_{i}$, and $\phi_{i}$ are normally distributed around zero, independent for each ion, and constant on the timescale of gate operations.

Figure 7 shows the infidelity and over- and underrotation error as a function of the strength of misadjustments. In particular, to generate the data shown in the figure, 40 samples of each of the dimensionless parameters $50 \delta \omega_{i} / \omega_{0, i}, \boldsymbol{\delta}_{i} / l_{0}$ where $l_{0}=\left(e^{2} / 4 \pi \epsilon_{0} m \omega_{x}^{2}\right)^{1 / 3}, \theta_{i}$, and $\phi_{i}$ are drawn from a Gaussian distribution with width $\sigma$.

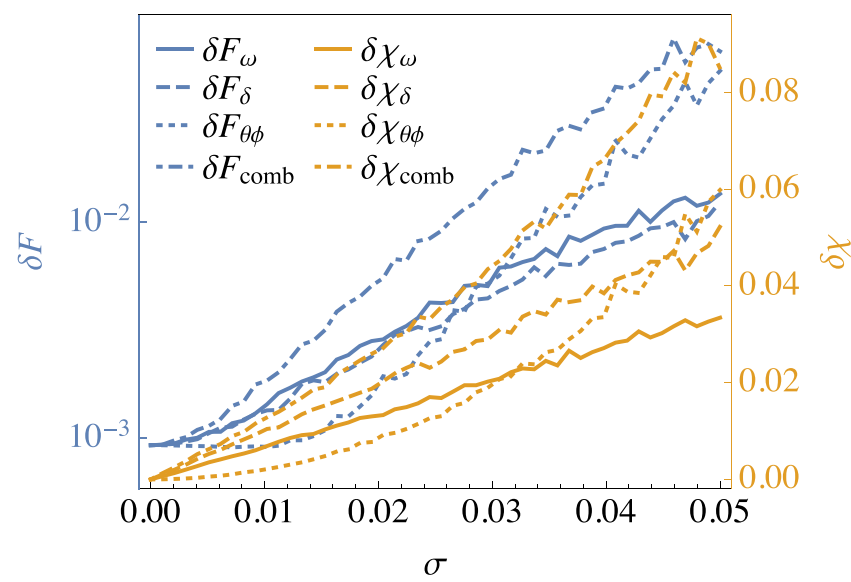

FIG. 7. Tweezer misadjustments. We show the infidelity Eq. (11) (blue) and the over- and under-rotation error Eq. (13) (orange) as a function of the strength of fluctuations $\sigma$ for three types of misadjustments and for their combination. For each type of misadjustment, the infidelity and the over- and under-rotation error are averaged over 40 realizations. We consider here a chain of 130 ions with 15 buffer ions on each side, and parameters $p=6, \epsilon \approx 0.07$, and $v_{0}=0.4$. 
The factor of 50 for shifts of the optical trapping frequency is introduced so that the infidelities and over- and under-rotation errors are comparable for all types of misadjustments on the range of values of $\sigma$ shown in Fig. 7. The assumption of those misadjustments being constant during the execution of a gate is motivated by the observation that beam pointing instabilities as well as laser intensity noise typically fall off rapidly above tens to hundreds of $\mathrm{Hz}[65,66]$. If we require $\delta F \lesssim 10^{-2}$ and $\delta \chi \lesssim 4 \times 10^{-2}$, this allows for standard deviations $\sigma$ of approximately 0.04 to 0.05 (the combination of all three types of misadjustments leads to the slightly more stringent requirement $\sigma \lesssim$ 0.02 to 0.03 ), which can be related to misadjustments of $70 \mathrm{~nm}$ for the tweezer focuses, $2^{\circ}$ for the incidence angles, and $10 \mathrm{kHz}$ for the pinning frequencies, corresponding to relative intensity errors of $3 \times 10^{-3}$ in a typical experiment with $d=10 \mu \mathrm{m}$ and $\omega_{x}=2 \pi \times 3 \mathrm{MHz}$. All three conditions can be satisfied in state-of-the-art experiments.

\section{E. Dynamical reconfiguration of tweezer arrays}

To perform consecutive layers of the quantum circuit shown in Fig. 1(d) the tweezer array has to be reconfigured dynamically. In particular, the second layer in the circuit in Fig. 1(d) can be implemented by switching off the tweezers that are focused on the ions that are affected by the gates $U_{1}, U_{2}$, and $U_{3}$, and by subsequently switching on optical tweezers focused on the equilibrium positions of the ions that are affected by the gates $U_{4}$ and $U_{5}$.

The dynamical reconfiguration of the optical tweezer array can cause heating by exciting phonon modes. Crucially, throughout the switching process, the phonon spectrum remains gapped, i.e., the smallest phonon frequency is larger than zero as in the right panel in Fig. 3(c), and heating is suppressed if the switching is performed adiabatically with respect to the phonon gap. Based on adiabatic perturbation theory, we derive conditions for adiabaticity for the worst-case scenario of an infinite ion chain in Appendix D. In this derivation, we assume for simplicity that all phonon modes are cooled to their ground state. We consider a switching protocol in which initially the first two ions within each unit cell of size $p$ are pinned, and at the end of the protocol the second and third ion are pinned. That is, during a time $\tau_{s}$, optical tweezers on the first and third ion are simultaneously switched off and on, respectively.

This process is adiabatic, i.e., the excitation of phonon modes is suppressed, if $\omega_{x} \tau_{s} \gg 8$ for $p=4$ and $\omega_{x} \tau_{s} \gg 11$ for $p=6$, for $\epsilon=0.07$ and $\nu_{0}=0.4$. Consequently, the switching time can be much shorter than the gate duration, which is $\omega_{x} \tau \approx 1400$ for gates for the same values of $\epsilon$ and $v_{0}$. Therefore, the total time it takes to execute a quantum circuit is dominated by the time for gate operations. Even shorter switching times are permissible for smaller values of $\nu_{0}$ and larger values of $\epsilon$.

\section{OPTIMIZED TWEEZER GATES}

The optimal choice of detunings and gate durations described above enables the implementation of parallel entangling tweezer gates in finite and infinite ion chains with a simple laser pulse. However, the density of the resulting quantum circuits is restricted by crosstalk. This limitation can be overcome and dense circuits as shown in Fig. 8 can be realized with techniques of optimal coherent control where gate operations are decomposed into multiple laser pulses.

\section{A. Infinite chains}

To optimize the operation of parallel entangling gates, we consider here the temporal modulation of the amplitudes of the laser pulses that drive the gates [18-25]. Originally, optimization of the amplitude shape was devised to implement fast gates with high fidelity. Our focus is on suppressing crosstalk in order to implement circuits with high density and fidelity. The control problem to be solved can be stated in terms of two conditions: the first condition reads $\chi_{i, i^{\prime}}=\chi_{i, i^{\prime}}^{0}$, where we set $\chi_{i, i^{\prime}}^{0}=-\pi / 4$ for $\left(i, i^{\prime}\right) \in I$ and $\chi_{i, i^{\prime}}^{0}=0$ for $\left(i, i^{\prime}\right) \in I^{\prime}$, and where the sets of pairs of ions $I$ and $I^{\prime}$ are defined as in Eqs. (7) and (12). The second condition, $\alpha_{i}^{n}=0$, ensures that there is no infidelity due to residual entanglement between qubits and phonon modes. To meet these conditions, we introduce as independent control parameters a variable number $S$ of pulse amplitudes $\Omega_{i}^{s}$ and detunings $\mu_{i}$ for ions $i \in\{1, \ldots, N\}$. Specifically, the laser pulse, which affects ion $i$ with detuning $\mu_{i}$, is divided into $S$ segments of equal duration with constant pulse amplitude $\Omega_{i}^{s}$ within a segment such that $\Omega_{i}(t)=$ $\Omega_{i}^{s}$ for $(s-1) \tau / S \leq t<s \tau / S$. If both of the above conditions are satisfied exactly, the implemented gate $U$ in Eq. (3) is identical to the ideal gate $U_{0}$ in Eq. (7). In practice, however, it is not possible to find exact solutions of
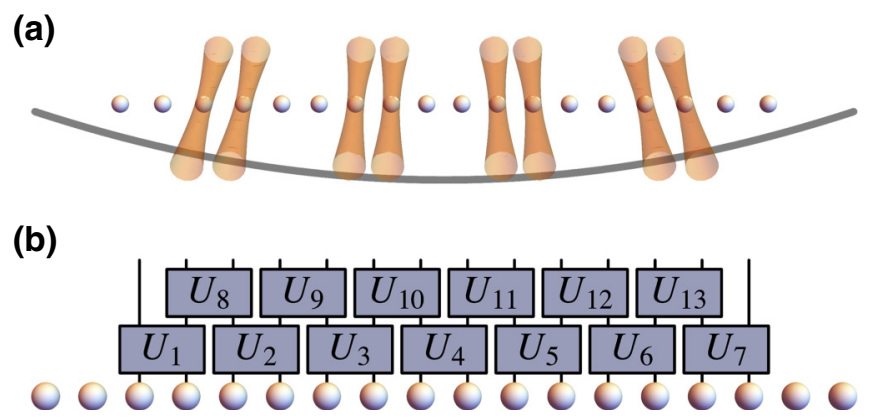

FIG. 8. Schematic setup for dense circuits. (a) In a setup in which pairs of ions that are pinned and not pinned alternate, gates can be performed on all ions in parallel. This leads to the realization of dense circuits as illustrated in (b). Maintaining high fidelity and low crosstalk in such circuits requires optimal coherent control techniques. 
this control problem. Instead, we search for approximate solutions by formulating the above conditions as an unconstrained optimization problem, i.e., we formulate a cost function $L$, which has to be minimized with respect to $\boldsymbol{\Omega}_{i}=\left(\Omega_{i}^{1}, \ldots, \Omega_{i}^{S}\right)$ and $\mu_{i}$ for given $\tau$. A minimum with $L=0$ would correspond to an exact solution of the control problem.

The definition of a cost function $L$ is not unique and we work with the choice $L=L_{\chi}+L_{\alpha}$, where

$$
L_{\alpha}=\sum_{i=1}^{N}\left(\sum_{n=1}^{N} \alpha_{i}^{n}\right)^{2}, \quad L_{\chi}=\sum_{\left(i, i^{\prime}\right) \in J}\left(\chi_{i, i^{\prime}}-\chi_{i, i^{\prime}}^{0}\right)^{2},
$$

and $J$ is a set of ion pairs, which is specified below. $L_{\chi}$ corresponds to the simplest polynomial in Rabi frequencies that has a minimum with $L_{\chi}=0$ at $\chi_{i, i^{\prime}}=\chi_{i, i^{\prime}}^{0}$. The square in the definition of $L_{\alpha}$ ensures that $L_{\alpha}$ is a polynomial of Rabi frequencies of the same order as $L_{\chi}$. More details can be found in Appendix B.

We consider now an infinite chain, which is subdivided by optical tweezers into unit cells of size $p=4$. Our goal is to perform entangling gates on both the set of pinned ions as well as the set of ions that are not pinned to realize a maximally dense quantum circuit as illustrated in Fig. 9. Crosstalk between these two sets of ions is suppressed through the small spatial overlap of the respective phonon modes. Therefore, we minimize the cost function for both sets independently, and we calculate the infidelity and crosstalk, which result from performing the independently optimized gates simultaneously a posteriori.

To suppress crosstalk within each set of ions, it is sufficient to allow for only a small number $G$ of independent sequences of Rabi frequencies $\boldsymbol{\Omega}_{i}$ : first, we choose the sequences of Rabi frequencies to be the same for two neighboring pinned or not pinned ions, i.e., we set $\boldsymbol{\Omega}_{2 i-1}=$ $\boldsymbol{\Omega}_{2 i}$. Second, since crosstalk is negligible for sufficiently distant pairs of ions, we limit the "active" suppression of crosstalk through the minimazition of $L$ to a group of $G$ neighboring pairs of ions within each subset. The resulting $G$ independent sequences $\boldsymbol{\Omega}_{i}$ are applied periodically in space, that is, we set $\boldsymbol{\Omega}_{i}=\boldsymbol{\Omega}_{i+4 G}$. Thus, for gates on pinned ions, the set $J$ in Eq. (23) contains pairs $\left(i, i^{\prime}\right)$ for which the first is any one of the pinned ions up to unit cell $G, i \in$ $\{1,2,5,6, \ldots, 4 G-3,4 G-2\}$ and $i^{\prime}$ runs over all ions to the right of $i$. For gates on ions that are not pinned, the first ion $i$ in $\left(i, i^{\prime}\right)$ is in the set $i \in\{3,4,7,8, \ldots, 4 G-1,4 G\}$ and again $i^{\prime}$ runs over all ions to the right of $i$.

The gate optimization for infinite systems is illustrated in Fig. 9 for $\omega_{x} \tau=1500, S=8$, and $G=4$. Figure 9(a) shows the mode spectrum for $p=4$ with COM and stretch bands both for the set of pinned and not pinned ions. We minimize $L$ independently for both sets of ions for detunings $\mu$ in ranges indicated by blue shaded areas.

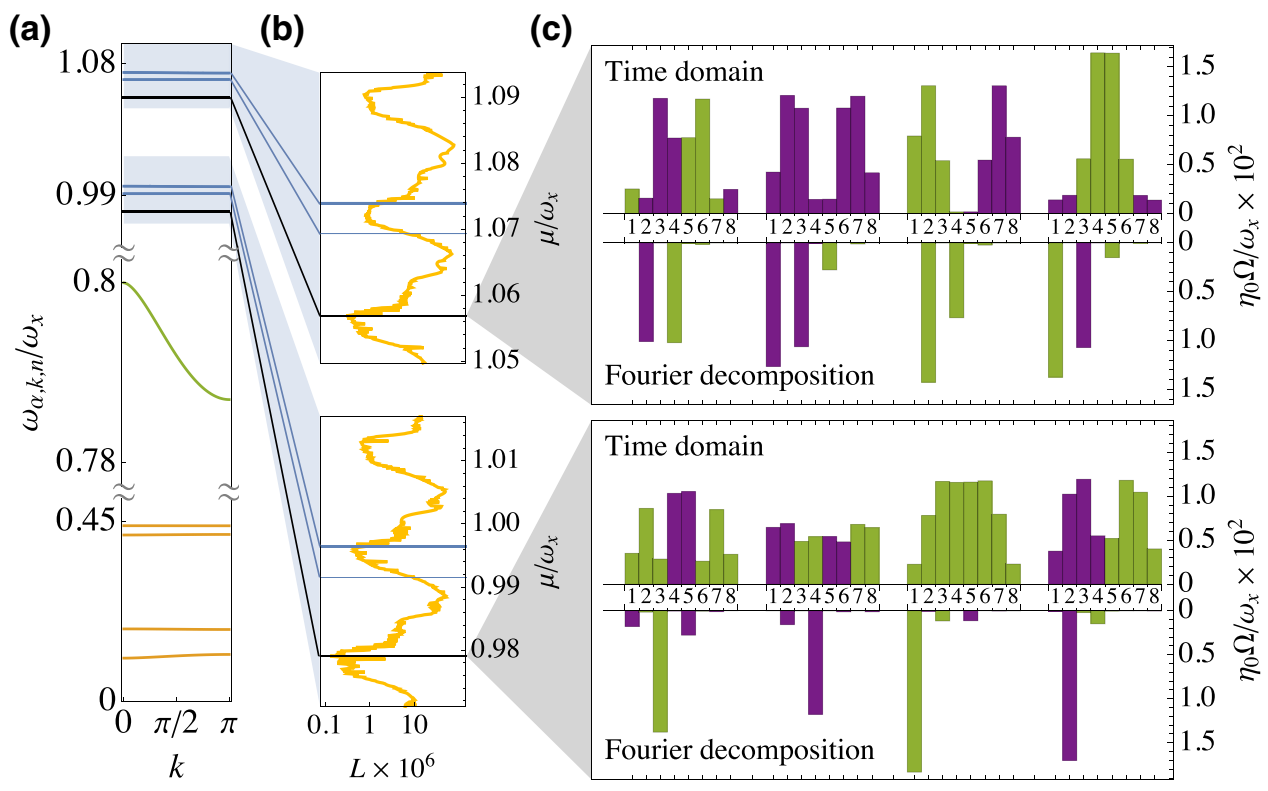

FIG. 9. Pulse optimization in an infinite chain. (a) Phonon-mode spectrum for $p=4, v_{0}=0.4$, and $\epsilon=0.07$. (b) The minimization of the cost function $L$ is performed independently for pinned and not pinned ions. The upper and lower panels show the cost function for optimal sequences of Rabi frequencies for a range of detunings around the COM and stretch bands of pinned and not pinned ions, respectively. (c) For the optimal detunings we show the corresponding $G=4$ independent sequences of $S=8$ Rabi frequencies both in the time domain and in discrete Fourier space. Purple and green correspond to positive and negative Rabi frequencies, respectively. The Fourier representations are dominated by few modes, which belong to subspaces of Fourier space that alternate along the chain: a pulse sequence that contains only odd Fourier components is followed by a sequence that is composed of even Fourier components and vice versa. 
Figure 9(b) shows the corresponding values of the cost function as the detuning is varied. The optimal detuning for each set is determined by the global minimum of $L$. Figure 9(c) shows the optimal sequences of Rabi frequencies both in the time domain and in discrete Fourier space. The Fourier representation is defined as

$$
\widetilde{\boldsymbol{\Omega}}=\mathrm{F} \boldsymbol{\Omega}, \quad \mathrm{F}_{s, s^{\prime}}=\frac{1}{\sqrt{S}} \sin \left[\pi s\left(s^{\prime}-1 / 2\right) / S\right],
$$

which corresponds to a discrete sine transform. Interestingly, the Fourier representations of the optimal pulse sequences are first of all dominated by few Fourier modes. Moreover, the dominant Fourier modes alternate along the ion chain, with a pulse sequence, which contains only odd Fourier components being followed by a sequence that is composed of even Fourier components and vice versa. This observation hints at a mechanism to suppress crosstalk, which is akin to refocusing circuits $[67,68]$. We stress that this mechanism is "discovered" here by an unbiased optimization algorithm.

If the independently optimized sets of gates are performed simultaneously, we find an average over- and under-rotation error and crosstalk per gate of $\delta \chi \approx 2.3 \times$ $10^{-7}$ and $C \approx 2.2 \times 10^{-3}$, respectively, and an average infidelity of $\delta F \approx 3.3 \times 10^{-4}$. While the infidelity is comparable to the results presented in Sec. IV A, crosstalk is significantly lower even though we consider here entangling gates acting on all ions in parallel. The values of the infidelity and crosstalk as well as the gate speed can be improved further by increasing the number of segments $S$ and the number of independent gates $G$. However, this leads to an increase of the required maximum Rabi frequency, which sets a limit on the achievable gate performance. At the same time, the complexity of the optimization problem as determined by the number of independent parameters grows linearly both with $S$ and $G$.

\section{B. Finite chains}

The results of the previous section show that optimal control can successfully be employed in order to implement dense and parallel tweezer gates in a periodic chain of equidistant ions. For this the periodicity of the system is a crucial requirement since then a small number of optimized pulse sequences can be repeated along the chain. We now introduce a method for finite chains that does not require periodicity. The central idea is to employ optimal control techniques given in Ref. [22] in order to independently optimize the infidelities for each tweezer gate and subsequently suppress crosstalk through the choice of laser detunings.

As in the previous section we divide the gate duration into $S$ segments with different but constant Rabi frequencies $\Omega_{i}^{s}$ for $s \in\{1 \ldots S\}$ and ions $i \in\{1 \ldots N\}$. The authors of Ref. [22] describe how to choose such a sequence of
Rabi frequencies in order to implement a single two-qubit gate for given gate duration and laser detuning and with minimal infidelity. Below we extend their ideas to dense quantum circuits of parallel tweezer gates as shown in Fig. 8(b). Our method works as follows: in a first step for each target ion pair $\left(i, i^{\prime}\right) \in I$ we determine a set of detunings $\mu$ in the vicinity of the corresponding localized modes for which an optimized pulse sequence yields an infidelity below a certain threshold value $\delta F_{\text {thresh }}$. Secondly we apply an iterative optimization algorithm to choose a detuning $\mu_{\left(i, i^{\prime}\right)}$ from each of these sets such that the total crosstalk becomes small for $\boldsymbol{\mu}=\left(\mu_{\left(i, i^{\prime}\right)}\right)_{\left(i, i^{\prime}\right) \in I}$. This is done by looping through the target pairs $I$ from the left end of the chain to the right end while applying the following routine: for the first pair, as well as in the case that for a given pair there is no detuning for which $\delta F<\delta F_{\text {thresh }}$, select the detuning that yields the lowest infidelity. Else select the detuning that minimizes crosstalk with all other pairs for which the detuning has already been fixed. Below we choose to iterate the optimization 5 times, which is sufficient to achieve convergence.

In Fig. 10 we show numerical results for a system of 130 ions with 15 buffer ions on each side. As in the infinite case we choose $p=4$ in order to implement a dense circuit consisting of 50 maximally entangling tweezer gates in

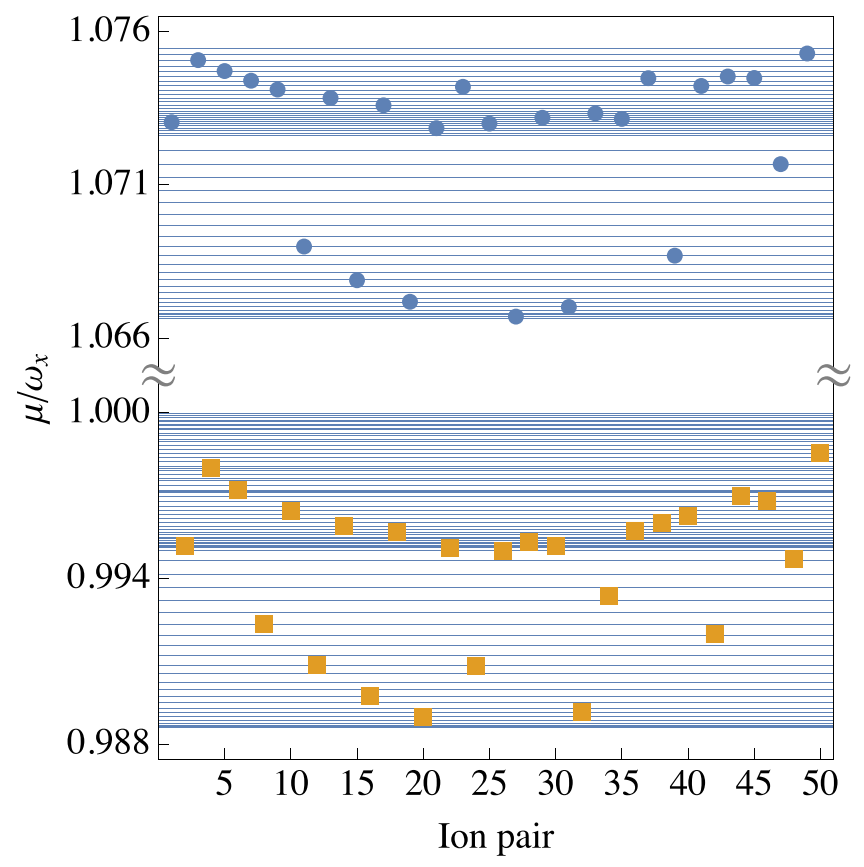

FIG. 10. Pulse optimization in a finite chain. We show the chosen detunings (see main text) to implement a dense circuit of 50 parallel, maximally entangling tweezer gates in a chain of 130 ions with 15 buffer ions on each side $\left(p=4, v_{0}=0.4, \epsilon \approx\right.$ $0.07)$. The horizontal lines mark the mode frequencies. We allow $S=8$ segments and set the gate duration to $\omega_{x} \tau=1500$, which requires a maximal Rabi frequency of $\eta_{0} \Omega / \omega_{x}=0.007$. We get average infidelity $\delta F=10^{-5}$ and crosstalk $C=2.77 \times 10^{-4}$. 
parallel, using individual optimal control and the iterative optimization as described above, where we set $\delta F_{\text {thresh }}=$ $10^{-3}$. As in the previous section we allow $S=8$ segments for each pulse sequence and we choose a gate duration of $\omega_{x} \tau=1500$. This yields an infidelity $\delta F=10^{-5}$ and a crosstalk $C=2.77 \times 10^{-4}$ for a maximal Rabi frequency of $\eta_{0} \Omega / \omega_{x}=0.007$. If higher Rabi frequencies are available one could increase the number of segments for the pulse sequences in order to further improve infidelity or to speed up the gate [22].

\section{OUTLOOK}

In this work, we develop the implementation of scalable parallel gate operations using localized transverse phonon modes generated by optical tweezers. To be concrete, we consider quantum circuits with spatially recurring structures of nearest-neighbor two-qubit gates as illustrated in Figs. 1(d) and 8(b). The dynamical reconfigurability of programmable tweezer arrays enables reshaping phonon modes on the fly. This is a key feature of optical segmentation of ion chains and facilitates the efficient implementation of universal parallelized quantum circuits.

Immediate extensions of the methods developed in this paper are illustrated schematically in Fig. 1(a). First, multiqubit gates can be performed on subregisters, which are separated by "optical tweezer walls" [34]; second, the COM and stretch modes of pairs of distant ions can be used to implement entangling gates for qubits, which are not nearest neighbors. Combining these capabilities leads to the realization of $1 \mathrm{D}$ quantum networks, which connect nodes that correspond to subregisters of long 1D chains.

Beyond these opportunities for quantum algorithms and gate-based digital quantum simulation, designer phonon modes, which are shaped through optical potentials open up new possibilities for analog quantum simulation, which can be realized through virtual far off-resonant excitation of phonon modes.

In addition to the applications of optical tweezers in the implementation of quantum gates and the design of Hamiltonians for analog quantum simulation, they also provide new possibilities to tackle challenges on a more fundamental level of quantum hardware design. For example, while we focus here on programming the phonon-mode structure for a given configuration of the ion chain, where the equilibrium positions of the ions are fixed by the trapping potential and Coulomb interactions, and tweezers are focused on the equilibrium positions, also the equilibrium positions themselves can be shifted by using optical forces. This enables, e.g., to achieve uniform ion spacings along the chain to facilitate individual control by addressed laser beams for gate operations, and thus provides an alternative to anharmonic potentials $[22,69]$. Further, laser cooling of phonon modes can be carried out more efficiently in an ion chain that is divided into subregisters [34].
An interesting question concerns the extension of the methods developed in this paper to two-dimensional and 3D structures [70-72]. Further studies are required to elucidate the interplay between micromotion [73,74], which is not negligible in specific spatial directions, and the localization properties of phonon modes in higher dimensions.

\section{ACKNOWLEDGMENTS}

We thank R. Islam and R. Blatt for insightful comments and helpful discussions. Theoretical work at Innsbruck is supported by the European Union program Horizon 2020 under Grants Agreement No. 817482 (PASQuanS) and No. 731473 (QuantERA via QTFLAG), the US Air Force Office of Scientific Research (AFOSR) via IOE Grant No. FA9550-19-1-7044 LASCEM, by the Simons Collaboration on Ultra-Quantum Matter, which is a grant from the Simons Foundation (651440, P.Z.), and by the Institut für Quanteninformation. L.P., P.S., and T.M. acknowledge support from the Austrian Science Fund (FWF) through the SFB BeyondC: F7102, and the IQI GmbH. P.S. acknowledges support from the Austrian Research Promotion Agency (FFG) contract 872766.

L.S. and P.Z. guided the research based on original ideas proposed by P.Z. L.S. and T.O. performed the analytical and numerical studies underlying the manuscript. L.P., P.S., and T.M. contributed the experimental feasibility study, and experimental perspective. The manuscript was written by L.S. supported by T.O. and P.Z. All authors contributed to the discussion of results.

\section{APPENDIX A: PHONON MODES IN 1D ION CHAINS WITH OPTICAL TWEEZERS}

In the following, we derive phonon-mode matrices $M_{\alpha, i}^{n}$ and mode frequencies $\omega_{\alpha, n}$ for finite and infinite 1D chains of trapped ions, which are subject to programmable arrays of optical tweezers.

\section{Phonon modes of finite chains}

The Hamiltonian for the classical 3D motion of $N$ ions in a harmonic trap reads

$$
H_{0}=\sum_{i=1}^{N}\left[\frac{p_{i}^{2}}{2 m}+V\left(\mathbf{r}_{i}\right)\right]+\frac{e^{2}}{4 \pi \epsilon_{0}} \sum_{i<i^{\prime}=1}^{N} \frac{1}{\left|\mathbf{r}_{i}-\mathbf{r}_{i^{\prime}}\right|},
$$

where $\mathbf{r}_{i}=\left(r_{x, i}, r_{y, i}, r_{z, i}\right)^{\top}=\left(x_{i}, y_{i}, z_{i}\right)^{\top}$ and $\mathbf{p}_{i}=\left(p_{x, i}, p_{y, i}\right.$, $\left.p_{z, i}\right)^{\top}$ are, respectively, the position and momentum of ion $i$, and the electronic trapping potential is given by $V(\mathbf{r})=\sum_{\alpha \in\{x, y, z\}} \frac{1}{2} m \omega_{\alpha} r_{\alpha}^{2}$. We assume tight confinement in the transverse $x$ and $y$ directions, such that the equilibrium positions $\mathbf{r}_{i, 0}$ of the ions are along the $z$ axis, $\mathbf{r}_{i, 0}=\left(0,0, z_{i, 0}\right)^{\top}$, and form a linear $1 \mathrm{D}$ chain. If the number of ions $N$ is increased, the axial trapping frequency $\omega_{z}$ 
has to be reduced for the linear configuration of the ion chain to remain stable [75-77].

Phonon modes of the ion chain correspond to quantized small-amplitude oscillations of the ions around their equilibrium positions. An expansion of the Hamiltonian Eq. (A1) to second order in $\delta \mathbf{r}_{i}=\mathbf{r}_{i}-\mathbf{r}_{i, 0}$ leads to $H_{0}=$ $\sum_{\alpha \in\{x, y, z\}} H_{0, \alpha}$ where

$$
\begin{aligned}
H_{0, \alpha}= & \sum_{i=1}^{N}\left(\frac{p_{\alpha, i}^{2}}{2 m}+\frac{1}{2} m \omega_{\alpha, i}^{2} \delta r_{\alpha, i}^{2}\right) \\
& +\frac{e^{2} s_{\alpha}}{4 \pi \epsilon_{0}} \sum_{i<i^{\prime}=1}^{N} \frac{\delta r_{\alpha, i} \delta r_{\alpha, i^{\prime}}}{\left|z_{i, 0}-z_{i^{\prime}, 0}\right|^{3}},
\end{aligned}
$$

with $s_{x}=s_{y}=1$ and $s_{z}=-2$. To this order of the expansion, oscillations of the ions in the transverse $x$ and $y$ directions and the longitudinal $z$ direction decouple. The ions perform harmonic oscillations around their equilibrium positions with local trapping frequencies

$$
\omega_{\alpha, i}^{2}=\omega_{\alpha}^{2}-\frac{e^{2} s_{\alpha}}{4 \pi \epsilon_{0} m} \sum_{\substack{i^{\prime}=1 \\ i^{\prime} \neq i}}^{N} \frac{1}{\left|z_{i, 0}-z_{i^{\prime}, 0}\right|^{3}} .
$$

These oscillations are coupled by the residual Coulomb interaction described by the last term in Eq. (A2). To design phonon modes, we consider adjusting the local trapping frequencies by focusing optical tweezers on the equilibrium positions of the ions as detailed in Sec. III. The optical potential, which is generated by the tweezers, is described by an additional contribution to the Hamiltonian that reads $H_{1}=\sum_{i=1}^{N} V_{i}^{\mathrm{twz}}\left(\mathbf{r}_{i}\right)$, where

$$
V_{i}^{\mathrm{twz}}\left(\mathbf{r}_{i}\right)=\frac{1}{2} m \omega_{0, i}^{2}\left(\delta r_{x, i}^{2}+\delta r_{z, i}^{2}\right) .
$$

Normal-mode coordinates $\xi_{\alpha, n}$ are introduced via the linear transformation $\delta r_{\alpha, i}=\sum_{n=1}^{N} M_{\alpha, i}^{n} \xi_{i}$, where $M_{\alpha, i}^{n}$ is the mode matrix, which diagonalizes the phonon Hamiltonian $H_{\mathrm{ph}}=H_{0}+H_{1}$, i.e., which brings the Hamiltonian to a form that corresponds to decoupled harmonic oscillators with frequencies $\omega_{\alpha, n}$. The normal-mode oscillations of the ion chain can be quantized by introducing annihilation and creation operators for phonons, $a_{\alpha, n}$ and $a_{\alpha, n}^{\dagger}$, respectively. In terms of these operators, the deviation of ion $i$ from its equilibrium position can be expressed as

$$
\delta r_{\alpha, i}=\sqrt{\frac{\hbar}{2 m}} \sum_{n=1}^{N} \frac{M_{\alpha, i}^{n}}{\sqrt{\omega_{\alpha, n}}}\left(a_{\alpha, n}+a_{\alpha, n}^{\dagger}\right) .
$$

The qubit-phonon Hamiltonian Eq. (1) couples the qubits, which are encoded in individual ions, to the quantized normal-mode oscillations of the ion chain.

\section{Phononic band structure of infinite chains}

We proceed to derive the phononic band structure for an infinitely long 1D ion chain with uniform spacing $d$. The ions are subject to a spatially periodic array of optical tweezers, which subdivides the ion chain into unit cells of size $p$. Within each unit cell, the first two ions are pinned by optical tweezers with trapping frequency $\omega_{0}$, and the remaining $p-2$ ions are not pinned as illustrated in Fig. 3(a).

We label the ions by their unit cell $l \in \mathbb{Z}$ and their position $i=1, \ldots, p$ within the unit cell, where the pinned ions correspond to the positions $i=1,2$. The classical Hamiltonian for small-amplitude oscillations of the ions around their respective equilibrium positions can be written as $H_{\mathrm{ph}}=\sum_{\alpha \in\{x, y, z\}} H_{\alpha}$, where $H_{\alpha}$ is given by the sum of Eq. (A2) and the optical potential in Eq. (A4) in the simultaneous limit $N \rightarrow \infty$ and $\omega_{z} \rightarrow 0$ :

$$
\begin{aligned}
H_{\alpha}= & \sum_{l \in \mathbb{Z}} \sum_{i=1}^{p}\left(\frac{p_{\alpha, l, i}^{2}}{2 m}+\frac{1}{2} m \widetilde{\omega}_{\alpha, i^{2}}^{2} \delta r_{\alpha, l, i}^{2}\right) \\
& +\frac{1}{2} s_{\alpha} \sum_{l, l^{\prime} \in \mathbb{Z}} \sum_{i, i^{\prime}=1}^{p} \delta r_{\alpha, l, i} C_{i-i^{\prime}}^{l-l^{\prime}} \delta r_{\alpha, l^{\prime}, i^{\prime}} .
\end{aligned}
$$

The local trapping frequency, which the ions at position $i$ within each unit cell experience, is given by

$$
\widetilde{\omega}_{\alpha, i}^{2}=\omega_{\alpha}^{2}-\frac{s_{\alpha} e^{2} \zeta(3)}{2 \pi \epsilon_{0} d^{3} m}+\omega_{\alpha, 0}^{2}\left(\delta_{i, 1}+\delta_{i, 2}\right),
$$

where, according to Eq. (A4), $\omega_{x, 0}=\omega_{z, 0}=\omega_{0}$ and $\omega_{y, 0}=$ 0 , and $\zeta(s)$ is the Riemann zeta function. The translationally invariant coupling coefficient $C_{i}^{l}$ reads

$$
C_{i}^{l}= \begin{cases}0 & \text { for } l=0 \text { and } i=0 \\ \frac{e^{2}}{4 \pi \epsilon_{0} d^{3}} \frac{1}{|p l+i|^{3}} & \text { else. }\end{cases}
$$

\section{a. Phononic band structure for periodic tweezer arrays}

We seek the normal-mode matrix, which diagonalizes the potential energy contribution to Eq. (A6). It is convenient to write the latter as $H_{\mathrm{pot}, \alpha}=\frac{1}{2} m \omega_{x}^{2} V_{\alpha}$, where $V_{\alpha}$ is dimensionless. For concreteness and to simplify the notation, we focus in the following on oscillations in the $x$ direction, and we omit the subscript $\alpha=x$. The normal modes of oscillations in the $y$ and $z$ directions can be found analogously.

To account for the translational invariance of the phonon Hamiltonian (A6) in the unit-cell index $l$, we interpret the coordinates $\delta x_{l, i}$ as coefficients of a Fourier series, $c_{k, i}=$ $\sum_{l \in \mathbb{Z}} e^{-i k l} \delta x_{l, i}$, where $k$ is analogous to the quasimomentum of an electron in a solid. In terms of the new complex 
coordinates $c_{k, i}$, the potential energy reads

$$
V=\int_{-\pi}^{\pi} \frac{d k}{2 \pi} \sum_{i, i^{\prime}=1}^{p} v_{i, i^{\prime}}^{k} c_{k, i}^{*} c_{k, i^{\prime}},
$$

where

$$
v_{i, i^{\prime}}^{k}=V_{i} \delta_{i, i^{\prime}}+J_{i-i^{\prime}}^{k}, \quad J_{i}^{k}=\sum_{l \in \mathbb{Z}} J_{i}^{l} e^{-i k l},
$$

with $\quad V_{i}=1-2 \epsilon^{2} \zeta(3)+v_{0}^{2}\left(\delta_{i, 1}+\delta_{i, 2}\right) \quad$ and $\quad J_{i}^{l}=C_{i}^{l} /$ $\left(m \omega_{x}^{2}\right)$. We next introduce new coordinates $b_{k, n}=$ $\sum_{i=1}^{p} B_{i}^{k, n *} c_{k, i}$, where $B_{i}^{k, n}$ is the unitary matrix that diagonalizes $v_{i, i^{\prime}}^{k}$ with eigenvalues $v_{k, n}=\omega_{k, n} / \omega_{x}$. While $V$ is diagonal in terms of the coordinates $b_{k, n}$, they cannot be interpreted as proper normal-mode coordinates because they are complex and not independent: since $\delta x_{l, i}$ are real, it follows that $b_{k, n}^{*}=b_{-k, n}$. Therefore, we restrict the range of values of the quasimomentum to $k \in[0, \pi]$, and we decompose $b_{k, n}$ and $B_{i}^{k, n}$ into real and imaginary parts, $b_{k, n}=1 / \sqrt{2}\left(\xi_{k, n, 1}+i \xi_{k, n, 2}\right)$ and $B_{i}^{k, n}=\Xi_{i}^{k, n, 1}+i \Xi_{i}^{k, n, 2}$, to obtain

$$
\delta x_{l, i}=\int_{0}^{\pi} \frac{d k}{2 \pi} \sum_{n=1}^{p} \sum_{\lambda=1}^{2} M_{l, i}^{k, n, \lambda} \xi_{k, n, \lambda} .
$$

$\xi_{k, n, \lambda}$ are the desired real and independent normal-mode coordinates, and the normal-mode transformation matrices are given by

$$
\begin{aligned}
& M_{l, i}^{k, n, 1}=\sqrt{2}\left[\cos (k l) \Xi_{i}^{k, n, 1}-\sin (k l) \Xi_{i}^{k, n, 2}\right], \\
& M_{l, i}^{k, n, 2}=-\sqrt{2}\left[\cos (k l) \Xi_{i}^{k, n, 2}+\sin (k l) \Xi_{i}^{k, n, 1}\right] .
\end{aligned}
$$

\section{b. Perturbative expansion for strong pinning}

For strong pinning $v_{0} \gg \epsilon$, the matrix $v^{k}$ can be diagonalized perturbatively in $J_{i}^{k} \propto \epsilon^{2}$. To zeroth order, we obtain two degenerate subspaces, which correspond to the ions that are pinned and not pinned. The respective eigenvalues are $1-2 \epsilon^{2} \zeta(3)+v_{0}^{2}$ and $1-2 \epsilon^{2} \zeta(3)$, and have degeneracy 2 and $p-2$. To obtain the leading corrections to the eigenvalues in degenerate perturbation theory, we omit the elements of $v^{k}$ that couple the degenerate subspaces, whereupon $v^{k}$ becomes block diagonal. The matrix $B^{k}$ that diagonalizes the $2 \times 2$ block of $v^{k}$ that describes the pinned ions, reads

$$
B^{k} \sim \Xi^{1}=\frac{1}{\sqrt{2}}\left(\begin{array}{cc}
1 & 1 \\
1 & -1
\end{array}\right),
$$

where we omit corrections proportional to $1 / p^{4}$, which are small for $p \gtrsim 4$. Evidently, for $p \rightarrow \infty$, the modes that form the highest two bands, are indeed COM and stretch modes of pairs of pinned ions within each unit cell. The corresponding $2 \times 2$ blocks of mode matrices in Eq. (A12) are given by $M_{l}^{k, 1}=\sqrt{2} \cos (k l) \Xi^{1}$ and $M_{l}^{k, 2}=-\sqrt{2} \sin (k l) \Xi^{1}$. Perturbative corrections to the mode matrices are of order $O\left(\epsilon^{2} / \nu_{0}^{2}\right)$. The mode frequencies of the COM and stretch bands are

$$
v_{k, n}=\sqrt{1-2 \epsilon^{2} \zeta(3)+v_{0}^{2}+J_{0}^{k} \pm\left|J_{1}^{k}\right|},
$$

where $n=1$ for the COM band and $n=2$ for the stretch band. The width of these bands is determined by terms in Eq. (A14), which depend on the quasimomentum $k$, i.e., by $J_{0}^{k} \pm\left|J_{1}^{k}\right|$. For $p \gtrsim 4$, we find

$$
\begin{aligned}
& J_{0}^{k}+\left|J_{1}^{k}\right| \sim \epsilon^{2}\left\{1+\frac{4}{p^{3}} \operatorname{Re}\left[\operatorname{Li}_{3}\left(e^{i k}\right)\right]\right\}, \\
& J_{0}^{k}-\left|J_{1}^{k}\right| \sim-\epsilon^{2}\left\{1+\frac{12}{p^{5}} \operatorname{Re}\left[\operatorname{Li}_{5}\left(e^{i k}\right)\right]\right\},
\end{aligned}
$$

where $\operatorname{Li}_{\alpha}(z)=\sum_{n=1}^{\infty} \frac{z^{n}}{n^{\alpha}}$ denotes the polylogarithm. That is, the widths of the COM and the stretch bands are suppressed as $1 / p^{3}$ and $1 / p^{5}$, respectively. For $p \rightarrow \infty$, these bands become flat with frequencies

$$
v_{n}=\sqrt{1-2 \epsilon^{2} \zeta(3)+v_{0}^{2} \pm \epsilon^{2}}
$$

In this limit, pairs of pinned ions are completely decoupled, and the corresponding modes are strictly local within unit cells.

\section{APPENDIX B: EXPERIMENTAL FEASIBILITY STUDY}

Here we study the experimental feasibility of the proposed scheme to perform parallel tweezer gates in a trapped-ion quantum device. We discuss various sources of experimental imperfections, both for optical and groundstate qubit encodings, and for realizations with different ionic species.

\section{Spontaneous scattering}

A significant contribution to the infidelity of tweezer gates is due to scattering of photons of the optical tweezers. In the following, we discuss how the choice of qubit encoding and wavelength of the optical tweezer lasers effects the spontaneous scattering rate and therefore the infidelity of the gate.

Our discussion is based on the following model for the interaction of an optical tweezer beam with a trapped ion: The dipole potential induced by the optical tweezer is 
given by [78]

$$
U_{\text {dip }}(\mathbf{r})=-\frac{1}{2 \epsilon_{0} c} \operatorname{Re}(\alpha) I(\mathbf{r}),
$$

where $\epsilon_{0}$ is the vacuum permittivity, $c$ is the speed of light, $I(\mathbf{r})$ is the intensity of the tweezer beam at position $\mathbf{r}$, and $\alpha$ is the polarizability of the internal state of the ion. In general, the polarizability is different for the two states that encode the qubit. Therefore, a question we have to address below is how to ensure that both qubit states experience the same optical potential.

The scattering rate in the center of the Gaussian tweezer beam with frequency $\omega$, for a transition with resonance frequency $\omega_{0}$ and linewidth $\Gamma$, is [78]

$$
\Gamma_{\mathrm{sc}}(\omega)=\frac{3 c^{2}}{\hbar \omega_{0}^{3}}\left(\frac{\omega}{\omega_{0}}\right)^{3}\left(\frac{\Gamma}{\omega_{0}-\omega}+\frac{\Gamma}{\omega_{0}+\omega}\right)^{2} \frac{P}{W_{0}^{2}},
$$

with the total light power $P$ and the beam waist $W_{0}$. For a tightly focused beam with wavelength $\lambda$, the beam waist is approximately given by [79]

$$
W_{0} \approx 0.41 \times \frac{\lambda}{\mathrm{NA}},
$$

where NA is the numerical aperture of the focusing optics. All following estimations are carried out assuming NA = 0.7 , as shown in Ref. [80] in an ion-trapping experiment. Scattering rates from different transitions are summed up, whereas their trap potential partially cancels if the the sign of the detuning $\omega_{0}-\omega$ is opposite.

For a given spontaneous scattering rate $\Gamma_{\mathrm{sc}}$ and gate duration $\tau$, the infidelity of the gate due to scattering of light can be estimated as [81]

$$
\delta F_{\mathrm{sc}}=\frac{3}{2} \Gamma_{\mathrm{sc}} \tau
$$

\section{a. Ground-state qubit encoding}

A first approach to ensure that both qubit states experience the same optical potential is to encode both of them in the ground state of the ion [4]. In this case, the ac Stark shift for $\pi$-polarized light is not state dependent, and the tweezer wavelength can be varied over a broad range. We consider the species ${ }^{24} \mathrm{Mg}^{+},{ }^{40} \mathrm{Ca}^{+}$, ${ }^{88} \mathrm{Sr}^{+},{ }^{138} \mathrm{Ba}^{+}$, and ${ }^{171} \mathrm{Yb}^{+}$, which are used in experiments targeting quantum-information processing [4].

For all investigated ionic species, we only consider contributions to the optical trapping and to spontaneous scattering from the $S$ to $P$ transitions with linewidths of several MHz [82]. In Fig. 11, we show the infidelity $\delta F_{\text {sc }}$ as a function of the tweezer wavelength. The infidelity exhibits a single minimum, which lies in between a regime at short wavelengths near the resonance and a regime at

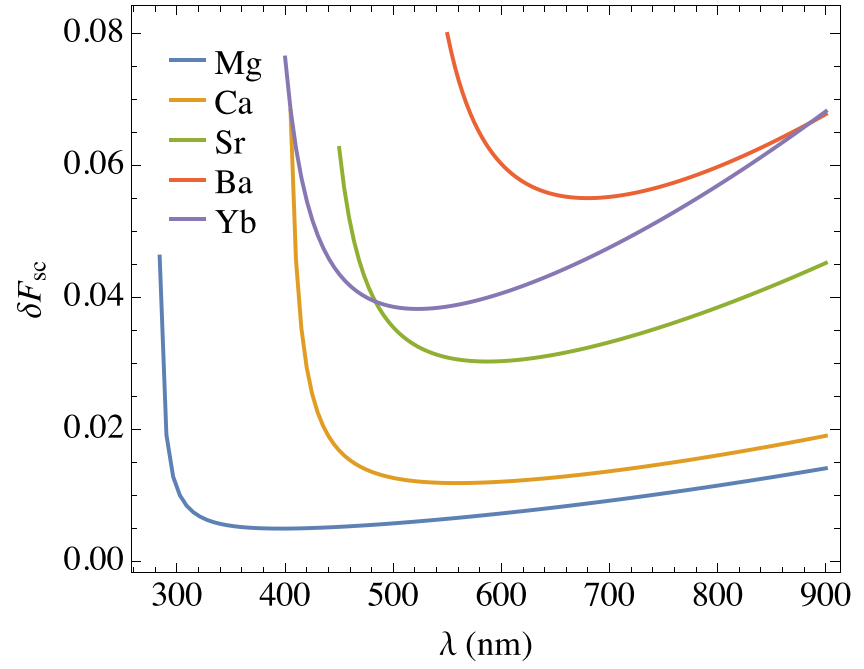

FIG. 11. Infidelity due to spontaneous scattering for $\epsilon=0.07$, $v_{0}=0.4$, and $\omega_{x}=2 \pi \times 3 \mathrm{MHz}$ for different atomic species. The steep slope at short wavelengths is caused by the excessive scattering close to the resonance of the $S \Leftrightarrow P$ transition. At long wavelengths, the infidelity increases due to an enlarged spot size for a diffraction limited spot for the assumed numerical aperture.

long wavelengths, for which the beam waist Eq. (B3) and, therefore, the required power to reach the strong pinning regime increases. Here we set $\epsilon=0.07$ and $\nu_{0}=0.4$ as in the examples considered in the main text. This results in $v_{0}^{2} / \epsilon^{2} \approx 32$, deeply in the regime of strong pinning. The gate duration is estimated according to Eqs. (A16) and (18).

The lowest infidelities can be achieved for ${ }^{24} \mathrm{Mg}^{+}$followed by ${ }^{40} \mathrm{Ca}^{+}$. For ${ }^{24} \mathrm{Mg}^{+}$ions in a trap with transverse trapping frequency $\omega_{x}=2 \pi \times 3 \mathrm{MHz}$ and a tweezer wavelength of $400 \mathrm{~nm}$, the contribution of spontaneous scattering to the infidelity is $\delta F_{\mathrm{sc}} \approx 4.9 \times 10^{-3}$. The corresponding interion distance is $d=15 \mu \mathrm{m}$. The infidelity caused by spontaneous scattering can be reduced by either increasing $d$ and decreasing $\omega_{x}$ (see Fig. 12), with the downside of increased heating rates [83], or by relaxing the condition on being in the strong trapping regime, which leads to higher infidelities from other sources (see Fig. 5). For $\epsilon=0.07$ and $\nu_{0}=0.2$, a scattering-induced infidelity of $\delta F_{\mathrm{sc}} \approx 1.2 \times 10^{-3}$ can be achieved. For the same values of $\omega_{x}, \epsilon$ and $\nu_{0}$, the infidelity for ${ }^{40} \mathrm{Ca}^{+}$ions is $2.8 \times 10^{-3}$ with $d=12.6 \mu \mathrm{m}$ and a tweezer wavelength of $532 \mathrm{~nm}$. The required power of the optical tweezers is on the order of a couple of $\mathrm{mW}$ for both species.

\section{b. Optical qubit encoding}

For an optical qubit, the qubit levels are encoded in two different electronic states. An apparent approach to ensure equal optical trapping potentials for both qubit states is to choose the tweezer wavelength as a magic wavelength with respect to the transition between the two qubit states. 


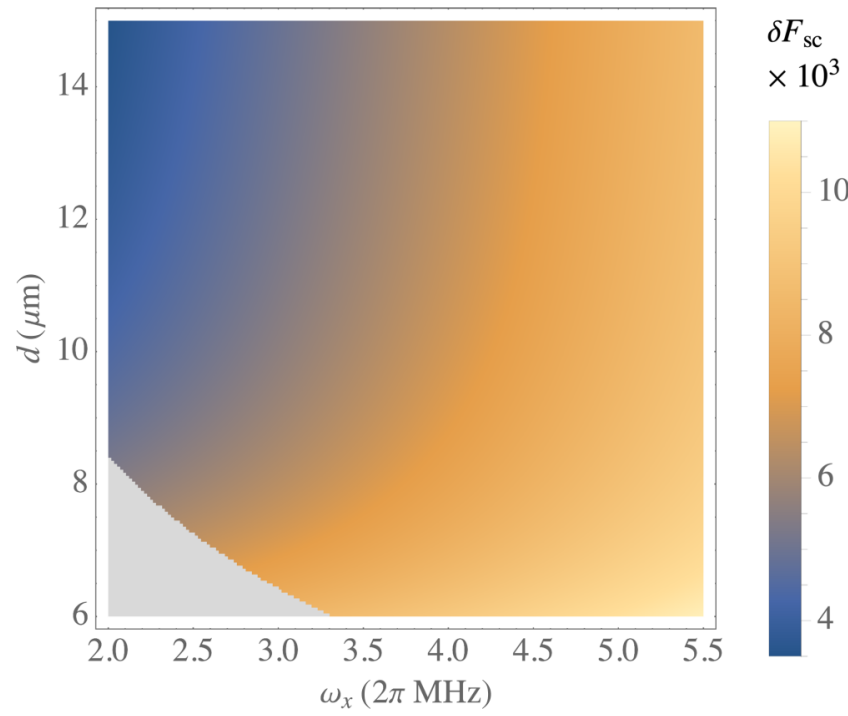

FIG. 12. Spontaneous scattering infidelity for parallel gates performed on ${ }^{24} \mathrm{Mg}^{+}$ground-state qubits with $400-\mathrm{nm}$ tweezer wavelength for varying ion distance $d$ and radial trap frequency $\omega_{x}$. By choosing $d$ and $\omega_{x}, \epsilon$ is fixed, and $\nu_{0}$ is determined in order to reach the strong pinning regime. The gray region marks parameter combinations that lead to a zigzag configuration of the ion string.

However, the polarizabities of ${ }^{40} \mathrm{Ca}^{+},{ }^{88} \mathrm{Sr}^{+}$, and ${ }^{138} \mathrm{Ba}^{+}$, which are commonly used species for optical qubits [4], are low at the respective magic wavelengths [84] and, therefore, high light intensities are required to achieve sizeable optical trapping potentials. Unfortunately, the concomitant increase of spontaneous scattering rates leads to relatively high infidelities.

In particular, magic wavelengths in between the $S_{1 / 2} \Leftrightarrow$ $P_{1 / 2}$ and the $S_{1 / 2} \Leftrightarrow P_{3 / 2}$ transitions have small detunings from broad transitions, which leads to scattering rates in the regime of hundreds of $\mathrm{kHz}$. Performing gates with typical gate durations $\tau$ on the order of tens to hundreds of $\mu \mathrm{s}$ is therefore rendered impossible.

For the abovementioned species, there also exists a set of magic wavelengths that is red detuned from all relevant transitions [84]. For ${ }^{40} \mathrm{Ca}^{+}$, this wavelength is at $1271 \mathrm{~nm}$ and therefore has a detuning of over $400 \mathrm{~nm}$ to the nearest relevant transition. Using tweezers at this magic wavelength yields lower scattering rates of hundreds of $\mathrm{Hz}$. However, the resulting infidelities are still in the $10^{-1}$ regime.

An alternative way to reduce the infidelity due to scattering is to use two superimposed tweezer beams at different wavelengths to trap each of the two qubit states individually. On the one hand, the gained flexibility with regard to the choice of wavelength could allow for low scattering rates while keeping the required power reasonable, but on the other hand additional experimental challenges would come into play. In particular, to avoid gate errors due to decoherence of the qubit induced by differential trapping potential fluctuations of the two qubit states, precise control over both the power in the tweezer beams as well as the positioning of the tweezers is required.

\section{Other experimental imperfections}

\section{a. Motional heating and cooling}

Another source of errors in entangling gates is a nonzero occupation of the phonon modes, which mediate the entangling interaction, as can be seen in Eq. (11). The occupation of phonon modes increases over time due to electric field noise. For $N$ ions in a single ion trap, the largest heating rate is proportional to $N$ for the COM mode where all ions oscillate in phase $[83,85]$. Ions that are pinned with optical tweezers, have localized phonon modes and, therefore, we expect that the heating rates for the two modes of each pinned pair are independent of the crystal size. We further expect that this holds also for the case where multiple gates are performed in parallel, since, due to the harmonic trapping potential, the localized modes have different mode frequencies as illustrated in Fig. 2, and thus the motion of distant pinned ions is not in phase.

For the proposed tweezer gates in long ion chains, the axial trapping frequency is kept very low to allow for interion distances of $10-15 \mu \mathrm{m}$. Without additional optical trapping, the spectrum of axial phonon modes reaches down to the axial trapping frequency. Since the motional heating rate is inversely proportional to the frequency, this low axial trapping frequency would result in a high phonon occupation in the axial modes of motion, leading to errors in entangling- and also single-qubit gates [86]. However, this effect is suppressed if several ions in the chain are pinned with optical tweezers, and, consequently, the lowest axial phonon frequency is shifted up as illustrated in Figs. 2 and 3. Moreover, since the oscillations of optically pinned and spectator ions decouple, gates on the pinned ions are only weakly affected by motional heating of phonon modes that are localized on spectator ions. Finally, the ability to split the ion chain into multiple subsets using the tweezer array can facilitate efficient cooling [34].

\section{b. Excess micromotion}

A trapped ion, which is not located in the rf null, gives rise to excess micromotion [87]. If the amplitude of this micromotion is comparable to the dimension of the optical tweezer, additional gate errors due to rf heating [88] and modulation of the optical potential are induced. The position of the ion and the rf null [89] can be overlapped much more precisely than the wavelength of the tweezer light and thus micromotion should not have an influence on the tweezer potential. 


\section{c. Differential ac Stark shift}

In Appendix B $1 \mathrm{a}$, we mention that for $\pi$-polarized light, the two qubit states encoded in ground states of an ion experience the same optical potential. If there is a contribution of circular polarization, a differential ac Stark shift between the two qubit states occurs. A temporal variation of this differential shift leads to dephasing of the qubit. Careful alignment and usage of high-quality polarization optics allow for a ratio of $10^{-5}$ of $\sigma$ - to $\pi$-polarized light. For a typical optical trap depth of $10 \mathrm{mK}$ and relative intensity fluctuation of the optical tweezer of $3 \times$ $10^{-3}$, as assumed in Sec. IV D, this results in differential ac-Stark-shift fluctuations of a couple of Hz. This should allow a gate fidelity of better than $99.5 \%$ [90].

\section{APPENDIX C: TWEEZER MISADJUSTMENTS}

In Sec. IV D in Eq. (22) we state the optical potential that is generated by a misadjusted optical tweezer. Here we describe how we calculate the effect of the shifts of the tweezer focuses. First note that since the optical potential is at most quadratic in the shifts of the tweezers, these shifts affect the modes of the ions only through the corresponding shifts in the equilibrium positions of the ions. We determine these shifts numerically up to second order in the shifts of the tweezers before averaging over several Gaussian realizations as before. To this end we expand the gradient of the total potential for a total number of $N$ ions

$$
\begin{aligned}
\nabla_{\xi} V= & \underbrace{\left.\sum_{m} \delta_{m}\left(\partial_{\delta_{m}} \nabla_{\xi} V\right)\right|_{\xi_{0}}}_{D} \\
& +\underbrace{\left.\frac{1}{2} \sum_{m, n} \delta_{m} \delta_{n}\left(\partial_{\delta_{m}} \partial_{\delta_{n}} \nabla_{\xi} V\right)\right|_{\xi_{0}}}_{W}
\end{aligned}
$$

where $\boldsymbol{\xi}=\boldsymbol{\xi}_{0}+X_{1} \boldsymbol{\delta}+\left(\begin{array}{c}\delta^{\top} X_{21} \delta \\ \delta^{\top} X_{22} \delta \\ \vdots\end{array}\right)$ with $\boldsymbol{\xi}_{0}=\left[x_{1}^{(0)}, x_{2}^{(0)}, \ldots\right.$ $\left.y_{1}^{(0)}, \ldots z_{N}^{(0)}\right]$ the equilibrium positions of the ions without tweezers, $\boldsymbol{\delta}=\left(\delta_{x, 1}, \delta_{x, 2}, \ldots \delta_{z, N}\right)$ the shifts of the tweezer focuses and $3 N \times 3 N$ matrices $X_{1},\left\{X_{2 m}\right\}_{m=1}^{3 N}$ that determine the new equilibrium positions from the shifts. From the condition $\nabla_{\xi} V=0$ to all orders we obtain

$$
D=0 \Leftrightarrow X_{1}=H^{-1} P,
$$

where $H_{i j}=\left.\left(\partial_{\xi_{i}} \partial_{\xi_{j}} V\right)\right|_{\xi_{0}}$ and $P_{i j}=\left.\left(\partial_{\xi_{i}} \partial_{\delta_{j}} V\right)\right|_{\xi_{0}}$. The second order yields

$$
W=0 \Leftrightarrow \mathbf{X}_{2}=H^{-1} \mathbf{b},
$$

where $\boldsymbol{X}_{2}=\left(X_{2 n}\right)_{n}$ and $\boldsymbol{b}=\left(-\frac{1}{2} X_{1}^{T} \mathcal{H}_{n} X_{1}\right)_{n}$ are vectors whose elements are matrices with $\mathcal{H}_{r}=\partial_{\xi r} H$. Hence the multiplication with $H^{-1}$ gives a linear combination of matrices for each element of $\boldsymbol{X}_{2}$.

\section{APPENDIX D: ADIABATIC SWITCHING OF TWEEZER ARRAYS}

Here we derive an estimate, based on adiabatic perturbation theory, for the excitation of phonons due to the switching of optical tweezers. We consider the switching protocol described by the phonon Hamiltonian (A6) with time-dependent local oscillation frequencies,

$$
\widetilde{\omega}_{\alpha, i}^{2}=\omega_{\alpha}^{2}-\frac{s_{\alpha} e^{2} \zeta(3)}{2 \pi \epsilon_{0} d^{3} m}+\omega_{\alpha, 0}^{2}\left[(1-s) \delta_{i, 1}+\delta_{i, 2}+s \delta_{i, 3}\right]
$$

which depend linearly on the parameter $s=t / \tau_{s}$ that varies between zero and one, and where $\tau_{s}$ denotes the switching time. At the beginning of the protocol at $s=0$, the ions at positions $i=1,2$ are pinned. At the end of the protocol, the optical potential on the ion at site $i=1$ is switched off, and the ions at positions $i=2,3$ are pinned.

The instantaneous eigenstates and energies of the phonon Hamiltonian obey $H_{\mathrm{ph}}(s)|n(s)\rangle=E_{n}(s)|n(s)\rangle$, where for simplicity we label the states with a single index $n \in \mathbb{N}_{0}$. To obtain an estimate of the excitation of phonons, we assume that all phonon modes are initially cooled to their ground state. The transition probability to an excited state $n>0$ is given by [91]

$$
P_{0 \rightarrow n} \sim \frac{1}{\tau_{s}^{2}}\left[\left.\frac{\left\langle n\left|\partial_{s} H_{\mathrm{ph}}\right| 0\right\rangle^{2}}{\left(E_{n}-E_{0}\right)^{4}}\right|_{s=0}+\left.\frac{\left\langle n\left|\partial_{s} H_{\mathrm{ph}}\right| 0\right\rangle^{2}}{\left(E_{n}-E_{0}\right)^{4}}\right|_{s=1}\right],
$$

and the total probability to excite phonon modes is $P=$ $\sum_{n \neq 0} P_{0 \rightarrow n}$. By evaluating the matrix elements of the phonon Hamiltonian explicitly, we find

$$
P=\frac{1}{2\left(\omega_{x} \tau_{s}\right)^{2}} \times \sum_{\alpha \in\{x, z\}} \int_{0}^{\pi} \frac{d k}{2 \pi} \sum_{n, n^{\prime}=1}^{p}\left(\left.A_{\alpha, k}^{n, n^{\prime}}\right|_{s=0}+\left.A_{\alpha, k}^{n, n^{\prime}}\right|_{s=1}\right),
$$

where, for $\alpha \in\{x, z\}$,

$$
A_{\alpha, k}^{n, n^{\prime}}=\frac{\omega_{x}^{2}}{\left(\omega_{\alpha, k, n}+\omega_{\alpha, k, n^{\prime}}\right)^{4}} \sum_{\lambda, \lambda^{\prime}=1}^{2}\left(c_{\alpha, \lambda, \lambda^{\prime}}^{k, n, n^{\prime}}\right)^{2}
$$

and

$$
c_{\alpha, \lambda, \lambda^{\prime}}^{k, n, n^{\prime}}=\frac{\omega_{0}^{2}}{2 \sqrt{\omega_{\alpha, k, n} \omega_{\alpha, k, n^{\prime}}}}\left(W_{\alpha, 3}^{\lambda, \lambda^{\prime}, k, n, n^{\prime}}-W_{\alpha, 1}^{\lambda, \lambda^{\prime}, k, n, n^{\prime}}\right),
$$


with

$$
\begin{aligned}
W_{\alpha, i}^{\lambda, \lambda, k, n, n^{\prime}} & =\Xi_{\alpha, i}^{k, n, 1} \Xi_{\alpha, i}^{k, n^{\prime}, 1}+\Xi_{\alpha, i}^{k, n, 2} \Xi_{\alpha, i}^{k, n^{\prime}, 2} \\
W_{\alpha, i}^{1,2, k, n, n^{\prime}} & =-W_{\alpha, i}^{2,1, k, n, n^{\prime}} \\
& =-\Xi_{\alpha, i}^{k, n, 1} \Xi_{\alpha, i}^{k, n^{\prime}, 2}+\Xi_{\alpha, i}^{k, n, 2} \Xi_{\alpha, i}^{k, n^{\prime}, 1} .
\end{aligned}
$$

These expressions yield the estimates stated in the main text in Sec. IV E.

\section{APPENDIX E: ENTANGLING GATES WITH TIME-MODULATED RABI FREQUENCIES}

Here we specialize Eqs. (5) and (6), which determine the entangling gate Eq. (3) to time-modulated Rabi frequencies [22].

\section{Finite chains}

The qubit-phonon coupling, Eq. (5), can be written as $\alpha_{i}^{n}=\mathbf{A}_{i}^{n} \cdot \mathbf{R}_{i}$, where the dimensionless Rabi frequency is given by $\mathbf{R}_{i}=\eta_{0} \boldsymbol{\Omega}_{i} / \omega_{x}=\left(R_{i}^{1}, \ldots, R_{i}^{S}\right)$ with the parameter $\eta_{0}$ defined in Eq. (20), and where $A_{i, s}^{n}=-i \sqrt{\omega_{x} / \omega_{n}} M_{i}^{n} g_{i}^{n, s}$ with $g_{i}^{n, s}=\omega_{x} \int_{(s-1) \tau / S}^{s \tau / S} d t \sin \left(\mu_{i} t\right) e^{i \omega_{n} t}$. Further, the qubitqubit coupling, Eq. (6), can be written as $\chi_{i, i^{\prime}}=\mathbf{R}_{i}^{\top} X_{i, i^{\prime}} \mathbf{R}_{i^{\prime}}$, where $X_{i, i^{\prime}}=\sum_{n=1}^{N}\left(\eta_{i}^{n} \eta_{i^{\prime}}^{n} / \eta_{0}^{2}\right) f_{i, i^{\prime}}^{n} . f_{i, i^{\prime}}^{n}$ is an $S \times S$ matrix with elements

$$
\begin{aligned}
f_{i, i^{\prime}}^{n, s, s^{\prime}}= & \omega_{x}^{2} \int_{(s-1) \tau / S}^{s \tau / S} d t \int_{\left(s^{\prime}-1\right) \tau / S}^{s^{\prime} \tau / S} d t^{\prime} \sin \left(\mu_{i} t\right) \sin \left(\mu_{i^{\prime}} t^{\prime}\right) \\
& \times \sin \left[\omega_{n}\left(t-t^{\prime}\right)\right] \quad \text { for } s>s^{\prime}
\end{aligned}
$$

$$
\begin{aligned}
f_{i, i^{\prime}}^{n, s, s^{\prime}}= & \omega_{x}^{2} \int_{(s-1) \tau / S}^{s \tau / S} d t \int_{(s-1) \tau / S}^{t} d t^{\prime}\left[\sin \left(\mu_{i} t\right) \sin \left(\mu_{i^{\prime}} t^{\prime}\right)\right. \\
& \left.+\sin \left(\mu_{i} t^{\prime}\right) \sin \left(\mu_{i^{\prime}} t\right)\right] \sin \left[\omega_{n}\left(t-t^{\prime}\right)\right] \quad \text { for } s=s^{\prime},
\end{aligned}
$$

$$
\begin{aligned}
f_{i, i^{\prime}}^{n, s, s^{\prime}}= & \omega_{x}^{2} \int_{\left(s^{\prime}-1\right) \tau / S}^{s^{\prime} \tau / S} d t \int_{(s-1) \tau / S}^{s \tau / S} d t^{\prime} \sin \left(\mu_{i} t^{\prime}\right) \sin \left(\mu_{i^{\prime}} t\right) \\
& \times \sin \left[\omega_{n}\left(t-t^{\prime}\right)\right] \quad \text { for } s<s^{\prime} .
\end{aligned}
$$

In terms of the vectors $\mathbf{A}_{i}^{n}$, the infidelity per gate Eq. (11) can be written as $\delta F=\frac{1}{G} \sum_{i=1}^{N} \mathbf{R}_{i}^{\top} \Phi_{i} \mathbf{R}_{i}$ where $\Phi_{i}^{s, s^{\prime}}=$ $\frac{8}{5} \sum_{n=1}^{N} \operatorname{Re}\left[\left(A_{i, s}^{n}\right)^{*} A_{i, s^{\prime}}^{n}\right]$.

\section{Infinite chains}

In infinite ion chains, the qubit-qubit coupling can be written as $\chi_{i, i^{\prime}}^{l, l^{\prime}}=\mathbf{R}_{l, i}^{\top} X_{i, i^{\prime}}^{l, l^{\prime}} \mathbf{R}_{l^{\prime}, i^{\prime}}$, where

$$
\begin{aligned}
X_{i, i^{\prime}}^{l, l^{\prime}}= & 2 \int_{0}^{\pi} \frac{d k}{2 \pi} \sum_{n=1}^{p} \sqrt{\frac{\omega_{x}}{\omega_{k, n}}} \\
& \times\left\{\cos \left[k\left(l-l^{\prime}\right)\right]\left[\Xi_{i}^{k, n, 1} \Xi_{i^{\prime}}^{k, n, 1}+\Xi_{i}^{k, n, 2} \Xi_{i^{\prime}}^{k, n, 2}\right]\right. \\
& \left.+\sin \left[k\left(l-l^{\prime}\right)\right]\left[\Xi_{i}^{k, n, 1} \Xi_{i^{\prime}}^{k, n, 2}-\Xi_{i}^{k, n, 2} \Xi_{i^{\prime}}^{k, n, 1}\right]\right\} f_{l, i, l^{\prime}, i^{\prime}}^{k, n}
\end{aligned}
$$

To obtain $X_{i, i^{\prime}}^{l, l^{\prime}}$, we approximate the integral over $k \in[0, \pi]$ by a discrete Riemann sum, and we calculate the matrices $\Xi_{i}^{k, n, \lambda}$ and the phonon-mode frequencies $\omega_{k, n}$ for each discrete value of $k$ numerically as described in Appendix A.

Similarly, for the matrix $\Phi_{l, i}$, which determines the infidelity per gate, we find

$$
\begin{aligned}
\Phi_{l, i}^{s, s^{\prime}}= & \frac{32}{5} \int_{0}^{\pi} \frac{d k}{2 \pi} \sum_{n=1}^{p} \sqrt{\frac{\omega_{x}}{\omega_{k, n}}} \sum_{\lambda=1}^{2}\left(\Xi_{i}^{k, n, \lambda}\right)^{2} \\
& \times \operatorname{Re}\left[\left(g_{l, i}^{k, n, s}\right)^{*} g_{l, i}^{k, n, s^{\prime}}\right] .
\end{aligned}
$$

[1] J. I. Cirac and P. Zoller, Quantum Computations with Cold Trapped Ions, Phys. Rev. Lett. 74, 4091 (1995).

[2] C. Gardiner and P. Zoller, in The Quantum World of UltraCold Atoms and Light Book II: The Physics of QuantumOptical Devices (World Scientific, Singapore, 2015), p. 1.

[3] P. Schindler, D. Nigg, T. Monz, J. T. Barreiro, E. Martinez, S. X. Wang, S. Quint, M. F. Brandl, V. Nebendahl, C. F. Roos, M. Chwalla, M. Hennrich, and R. Blatt, A quantum information processor with trapped ions, New J. Phys. 15, 123012 (2013).

[4] C. D. Bruzewicz, J. Chiaverini, R. McConnell, and J. M. Sage, Trapped-ion quantum computing: Progress and challenges, Appl. Phys. Rev. 6, 021314 (2019).

[5] C. J. Ballance, T. P. Harty, N. M. Linke, M. A. Sepiol, and D. M. Lucas, High-Fidelity Quantum Logic Gates Using Trapped-Ion Hyperfine Qubits, Phys. Rev. Lett. 117, 060504 (2016).

[6] Y. Shapira, R. Shaniv, T. Manovitz, N. Akerman, and R. Ozeri, Robust Entanglement Gates for Trapped-Ion Qubits, Phys. Rev. Lett. 121, 180502 (2018).

[7] V. Negnevitsky, M. Marinelli, K. K. Mehta, H. Y. Lo, C. Flühmann, and J. P. Home, Repeated multi-qubit readout and feedback with a mixed-species trapped-ion register, Nature 563, 527 (2018).

[8] T. P. Harty, D. T. C. Allcock, C. J. Ballance, L. Guidoni, H. A. Janacek, N. M. Linke, D. N. Stacey, and D. M. Lucas, High-Fidelity Preparation, Gates, Memory, and Readout of a Trapped-Ion Quantum Bit, Phys. Rev. Lett. 113, 220501 (2014). 
[9] J. P. Gaebler, T. R. Tan, Y. Lin, Y. Wan, R. Bowler, A. C. Keith, S. Glancy, K. Coakley, E. Knill, D. Leibfried, and D. J. Wineland, High-Fidelity Universal Gate Set for ${ }^{9} \mathrm{Be}^{+}$Ion Qubits, Phys. Rev. Lett. 117, 060505 (2016).

[10] K. Wright et al., Benchmarking an 11-qubit quantum computer, Nat. Commun. 10, 1 (2019).

[11] A. Erhard, H. P. Nautrup, M. Meth, L. Postler, R. Stricker, M. Ringbauer, P. Schindler, H. J. Briegel, R. Blatt, N. Friis, and T. Monz, Entangling logical qubits with lattice surgery, arXiv:2006.03071 (2020).

[12] Y. Lu, S. Zhang, K. Zhang, W. Chen, Y. Shen, J. Zhang, J. N. Zhang, and K. Kim, Global entangling gates on arbitrary ion qubits, Nature 572, 363 (2019).

[13] D. Zhu, N. M. Linke, M. Benedetti, K. A. Landsman, N. H. Nguyen, C. H. Alderete, A. Perdomo-Ortiz, N. Korda, A. Garfoot, C. Brecque, L. Egan, O. Perdomo, and C. Monroe, Training of quantum circuits on a hybrid quantum computer, Sci. Adv. 5, eaaw9918 (2019).

[14] Y. Wan, D. Kienzler, S. D. Erickson, K. H. Mayer, T. R. Tan, J. J. Wu, H. M. Vasconcelos, S. Glancy, E. Knill, D. J. Wineland, A. C. Wilson, and D. Leibfried, Quantum gate teleportation between separated qubits in a trapped-ion processor, Science 364, 875 (2019).

[15] D. F. V. James, Quantum dynamics of cold trapped ions with application to quantum computation, Appl. Phys. B: Lasers Opt. 66, 181 (1998).

[16] J. P. Schiffer, Phase Transitions in Anisotropically Confined Ionic Crystals, Phys. Rev. Lett. 70, 818 (1993).

[17] S. L. Zhu, C. Monroe, and L. M. Duan, Trapped ion Quantum Computation with Transverse Phonon Modes, Phys. Rev. Lett. 97, 050505 (2006).

[18] S.-L. Zhu, C. Monroe, and L.-M. Duan, Arbitrary-speed quantum gates within large ion crystals through minimum control of laser beams, Europhys. Lett. 73, 485 (2006).

[19] C. F. Roos, Ion trap quantum gates with amplitudemodulated laser beams, New J. Phys. 10, 013002 (2008).

[20] T. Choi, S. Debnath, T. A. Manning, C. Figgatt, Z.-X. Gong, L.-M. Duan, and C. Monroe, Optimal Quantum Control of Multimode Couplings between Trapped Ion Qubits for Scalable Entanglement, Phys. Rev. Lett. 112, 190502 (2014).

[21] S. Debnath, N. M. Linke, C. Figgatt, K. A. Landsman, K. Wright, and C. Monroe, Demonstration of a small programmable quantum computer with atomic qubits, Nature 536, 63 (2016).

[22] Y. Wu, S.-T. Wang, and L.-M. Duan, Noise analysis for high-fidelity quantum entangling gates in an anharmonic linear Paul trap, Phys. Rev. A 97, 062325 (2018).

[23] K. A. Landsman, Y. Wu, P. H. Leung, D. Zhu, N. M. Linke, K. R. Brown, L. Duan, and C. Monroe, Two-qubit entangling gates within arbitrarily long chains of trapped ions, Phys. Rev. A 100, 022332 (2019).

[24] C. Figgatt, A. Ostrander, N. M. Linke, K. A. Landsman, D. Zhu, D. Maslov, and C. Monroe, Parallel entangling operations on a universal ion-trap quantum computer, Nature 572, 368 (2019).

[25] Y. Lu, S. Zhang, K. Zhang, W. Chen, Y. Shen, J. Zhang, J.-N. Zhang, and K. Kim, Global entangling gates on arbitrary ion qubits, Nature 572, 363 (2019).

[26] P. H. Leung, K. A. Landsman, C. Figgatt, N. M. Linke, C. Monroe, and K. R. Brown, Robust 2-Qubit Gates in a
Linear Ion Crystal Using a Frequency-Modulated Driving Force, Phys. Rev. Lett. 120, 020501 (2018).

[27] D. Kielpinski, C. Monroe, and D. J. Wineland, Architecture for a large-scale ion-trap quantum computer, Nature 417, 709 (2002).

[28] H. Kaufmann, T. Ruster, C. T. Schmiegelow, M. A. Luda, V. Kaushal, J. Schulz, D. von Lindenfels, F. Schmidt-Kaler, and U. G. Poschinger, Scalable Creation of Long-Lived Multipartite Entanglement, Phys. Rev. Lett. 119, 150503 (2017).

[29] R. B. Blakestad, C. Ospelkaus, A. P. VanDevender, J. M. Amini, J. Britton, D. Leibfried, and D. J. Wineland, High-Fidelity Transport of Trapped-Ion Qubits through an X-Junction Trap Array, Phys. Rev. Lett. 102, 153002 (2009).

[30] J. M. Pino, J. M. Dreiling, C. Figgatt, J. P. Gaebler, S. A. Moses, M. S. Allman, C. H. Baldwin, M. Foss-Feig, D. Hayes, K. Mayer, C. Ryan-Anderson, and B. Neyenhuis, Demonstration of the QCCD trapped-ion quantum computer architecture, arXiv:2003.01293 (2020).

[31] D. Leibfried, R. Blatt, C. Monroe, and D. Wineland, Quantum dynamics of single trapped ions, Rev. Mod. Phys. 75, 281 (2003).

[32] A. Omran, H. Levine, A. Keesling, G. Semeghini, T. T. Wang, S. Ebadi, H. Bernien, A. S. Zibrov, H. Pichler, S. Choi, J. Cui, M. Rossignolo, P. Rembold, S. Montangero, T. Calarco, M. Endres, M. Greiner, V. Vuletić, and M. D. Lukin, Generation and manipulation of schrödinger cat states in rydberg atom arrays, Science 365, 570 (2019).

[33] D. Barredo, V. Lienhard, S. de Léséleuc, T. Lahaye, and A. Browaeys, Synthetic three-dimensional atomic structures assembled atom by atom, Nature 561, 79 (2018).

[34] Y.-C. Shen and G.-D. Lin, Scalable quantum computing stabilised by optical tweezers on an ion crystal, New J. Phys. 22, 053032 (2020).

[35] J. Loye, J. Lages, and D. L. Shepelyansky, Properties of phonon modes of an ion-trap quantum computer in the Aubry phase, Phys. Rev. A 101, 032349 (2020).

[36] P. A. Ivanov, S. S. Ivanov, N. V. Vitanov, A. Mering, M. Fleischhauer, and K. Singer, Simulation of a quantum phase transition of polaritons with trapped ions, Phys. Rev. A 80, 060301(R) (2009).

[37] A. Abdelrahman, O. Khosravani, M. Gessner, A. Buchleitner, H. P. Breuer, D. Gorman, R. Masuda, T. Pruttivarasin, M. Ramm, P. Schindler, and H. Häffner, Local probe of single phonon dynamics in warm ion crystals, Nat. Commun. 8, 1 (2017).

[38] D. Barredo, V. Lienhard, P. Scholl, S. de Léséleuc, T. Boulier, A. Browaeys, and T. Lahaye, Three-Dimensional Trapping of Individual Rydberg Atoms in Ponderomotive Bottle Beam Traps, Phys. Rev. Lett. 124, 023201 (2020).

[39] C. Schneider, M. Enderlein, T. Huber, and T. Schaetz, Optical trapping of an ion, Nat. Photon. 4, 772 (2010).

[40] E. A. Martinez, C. A. Muschik, P. Schindler, D. Nigg, A. Erhard, M. Heyl, P. Hauke, M. Dalmonte, T. Monz, P. Zoller, and R. Blatt, Real-time dynamics of lattice gauge theories with a few-qubit quantum computer, Nature 534, 516 (2016).

[41] J. T. Barreiro, M. Möller, P. Schindler, D. Nigg, T. Monz, M. Chwalla, M. Hennrich, C. F. Roos, P. Zoller, and R. 
Blatt, An open-system quantum simulator with trapped ions, Nature 470, 486 (2011).

[42] B. P. Lanyon, C. Hempel, D. Nigg, M. Müller, R. Gerritsma, F. Zähringer, P. Schindler, J. T. Barreiro, M. Rambach, G. Kirchmair, M. Hennrich, P. Zoller, R. Blatt, and C. F. Roos, Universal digital quantum simulation with trapped ions, Science 334, 57 (2011).

[43] F. Arute et al., Quantum supremacy using a programmable superconducting processor, Nature 574, 505 (2019).

[44] A. Nahum, J. Ruhman, S. Vijay, and J. Haah, Quantum Entanglement Growth under Random Unitary Dynamics, Phys. Rev. X 7, 031016 (2017).

[45] A. Nahum, S. Vijay, and J. Haah, Operator Spreading in Random Unitary Circuits, Phys. Rev. X 8, 21014 (2018).

[46] C. W. von Keyserlingk, T. Rakovszky, F. Pollmann, and S. L. Sondhi, Operator Hydrodynamics, OTOCs, and Entanglement Growth in Systems Without Conservation Laws, Phys. Rev. X 8, 21013 (2018).

[47] T. Rakovszky, F. Pollmann, and C. W. von Keyserlingk, Diffusive Hydrodynamics of Out-Of-Time-Ordered Correlators with Charge Conservation, Phys. Rev. X 8, 31058 (2018).

[48] V. Khemani, A. Vishwanath, and D. A. Huse, Operator Spreading and the Emergence of Dissipative Hydrodynamics under Unitary Evolution with Conservation Laws, Phys. Rev. X 8, 031057 (2018).

[49] G. J. Milburn, Simulating nonlinear spin models in an ion trap, arXiv:quant-ph/9908037 (1999).

[50] K. Mølmer and A. Sørensen, Multiparticle Entanglement of Hot Trapped Ions, Phys. Rev. Lett. 82, 1835 (1999).

[51] A. Sørensen and K. Mølmer, Quantum Computation with Ions in Thermal Motion, Phys. Rev. Lett. 82, 1971 (1999).

[52] A. Sørensen and K. Mølmer, Entanglement and quantum computation with ions in thermal motion, Phys. Rev. A 62, 022311 (2000).

[53] S.-L. Zhu and Z. D. Wang, Unconventional Geometric Quantum Computation, Phys. Rev. Lett. 91, 187902 (2003).

[54] J. J. García-Ripoll, P. Zoller, and J. I. Cirac, Coherent control of trapped ions using off-resonant lasers, Phys. Rev. A 71, 062309 (2005).

[55] C. Monroe, W. C. Campbell, L. M. Duan, Z. X. Gong, A. V. Gorshkov, P. Hess, R. Islam, K. Kim, G. Pagano, P. Richerme, C. Senko, and N. Y. Yao, Programmable Quantum Simulations of Spin Systems with Trapped Ions, arXiv:1912.07845 (2019).

[56] B. Kraus and J. I. Cirac, Optimal creation of entanglement using a two-qubit gate, Phys. Rev. A 63, 062309 (2001).

[57] M. A. Nielsen and I. L. Chuang, Quantum Computation and Quantum Information: 10th Anniversary Edition (Cambridge University Press, Cambridge, 2011), 10th ed.

[58] M. A. Nielsen, A simple formula for the average gate fidelity of a quantum dynamical operation, Phys. Lett. A 303, 249 (2002).

[59] I. Bengtsson and K. Zyczkowski, Geometry of Quantum States (Cambridge University Press, Cambridge, 2006).

[60] Y. R. Sanders, J. J. Wallman, and B. C. Sanders, Bounding quantum gate error rate based on reported average fidelity, New J. Phys. 18, 012002 (2015).

[61] R. Kueng, D. M. Long, A. C. Doherty, and S. T. Flammia, Comparing Experiments to the Fault-Tolerance Threshold, Phys. Rev. Lett. 117, 170502 (2016).
[62] D. Aharonov, A. Kitaev, and N. Nisan, in Proc. thirtieth Annu. ACM Symp. Theory Comput. - STOC '98 (ACM Press, New York, New York, USA, 1998), Vol. 1, p. 20.

[63] L. M. Duan, Scaling ion Trap Quantum Computation through Fast Quantum Gates, Phys. Rev. Lett. 93, 100502 (2004).

[64] H.-K. Li, E. Urban, C. Noel, A. Chuang, Y. Xia, A. Ransford, B. Hemmerling, Y. Wang, T. Li, H. Häffner, and X. Zhang, Realization of Translational Symmetry in Trapped Cold ion Rings, Phys. Rev. Lett. 118, 053001 (2017).

[65] T. Kanai, A. Suda, S. Bohman, M. Kaku, S. Yamaguchi, and K. Midorikawa, Pointing stabilization of a high-repetitionrate high-power femtosecond laser for intense few-cycle pulse generation, Appl. Phys. Lett. 92, 061106 (2008).

[66] F. Seifert, P. Kwee, M. Heurs, B. Willke, and K. Danzmann, Laser power stabilization for second-generation gravitational wave detectors, Opt. Lett. 31, 2000 (2006).

[67] V. Nebendahl, H. Häffner, and C. F. Roos, Optimal control of entangling operations for trapped-ion quantum computing, Phys. Rev. A 79, 012312 (2009).

[68] M. Müller, K. Hammerer, Y. L. Zhou, C. F. Roos, and P. Zoller, Simulating open quantum systems: From manybody interactions to stabilizer pumping, New J. Phys. 13, 085007 (2011).

[69] G.-D. Lin, S.-L. Zhu, R. Islam, K. Kim, M.-S. Chang, S. Korenblit, C. Monroe, and L.-M. Duan, Large-scale quantum computation in an anharmonic linear ion trap, Europhys. Lett. 86, 60004 (2009).

[70] W. M. Itano, J. J. Bollinger, J. N. Tan, B. Jelenković, X.-P. Huang, and D. J. Wineland, Bragg diffraction from crystallized Ion plasmas, Science 279, 686 (1998).

[71] M. Drewsen, C. Brodersen, L. Hornekær, J. S. Hangst, and J. P. Schiffer, Large Ion Crystals in a Linear Paul Trap, Phys. Rev. Lett. 81, 2878 (1998).

[72] A. Mortensen, E. Nielsen, T. Matthey, and M. Drewsen, Observation of Three-Dimensional Long-Range Order in Small Ion Coulomb Crystals in an rf Trap, Phys. Rev. Lett. 96, 103001 (2006).

[73] J. I. Cirac, L. J. Garay, R. Blatt, A. S. Parkins, and P. Zoller, Laser cooling of trapped ions: The influence of micromotion, Phys. Rev. A 49, 421 (1994).

[74] S. T. Wang, C. Shen, and L. M. Duan, Quantum computation under micromotion in a planar ion crystal, Sci. Rep. 5, 8555 (2015).

[75] S. Fishman, G. De Chiara, T. Calarco, and G. Morigi, Structural phase transitions in low-dimensional ion crystals, Phys. Rev. B 77, 064111 (2008).

[76] E. Shimshoni, G. Morigi, and S. Fishman, Quantum Zigzag Transition in Ion Chains, Phys. Rev. Lett. 106, 010401 (2011).

[77] J. Welzel, F. Stopp, and F. Schmidt-Kaler, Spin and motion dynamics with zigzag ion crystals in transverse magnetic gradients, J. Phys. B At. Mol. Opt. Phys. 52, 025301 (2019).

[78] R. Grimm, M. Weidemüller, and Y. B. Ovchinnikov, Optical Dipole Traps for Neutral Atoms, (Academic Press, London, 2000), p. 95.

[79] M. Bass, C. DeCusatis, J. Enoch, V. Lakshminarayanan, G. Li, C. Macdonald, V. Mahajan, and E. Van Stryland, Handbook of Optics, Volume II: Design, Fabrication and Testing, Sources and Detectors, Radiometry and Photometry (McGraw-Hill, Inc., New York, 2009). 
[80] G. Araneda, G. Cerchiari, D. B. Higginbottom, P. Holz, K. Lakhmanskiy, P. Obšil, Y. Colombe, and R. Blatt, The Panopticon device: an integrated Paul-trap-hemispherical mirror system for quantum optics, arXiv:2006.04828 (2020).

[81] C. J. Ballance, High-Fidelity Quantum Logic in $\mathrm{Ca}^{+}$ (Springer, New York, 2017).

[82] J. E. Sansonetti and W. C. Martin, Handbook of basic atomic spectroscopic data, J. Phys. Chem. Ref. Data 34, 1559 (2005).

[83] M. Brownnutt, M. Kumph, P. Rabl, and R. Blatt, Ion-trap measurements of electric-field noise near surfaces, Rev. Mod. Phys. 87, 1419 (2015).

[84] J. Kaur, S. Singh, B. Arora, and B. K. Sahoo, Magic wavelengths in the alkaline-earth-metal ions, Phys. Rev. A 92, 031402(R) (2015).

[85] M. K. Joshi, A. Fabre, C. Maier, T. Brydges, D. Kiesenhofer, H. Hainzer, R. Blatt, and C. F. Roos, Polarizationgradient cooling of 1D and 2D ion Coulomb crystals, New J. Phys. 22, 103013 (2020).

[86] M. Cetina, L. N. Egan, C. A. Noel, M. L. Goldman, A. R. Risinger, D. Zhu, D. Biswas, and C. Monroe,
Quantum Gates on Individually-Addressed Atomic Qubits Subject to Noisy Transverse Motion, arXiv:2007.06768 (2020).

[87] A. Mohammadi, J. Wolf, A. Krükow, M. Deiß, and J. Hecker Denschlag, Minimizing rf-induced excess micromotion of a trapped ion with the help of ultracold atoms, Appl. Phys. B 125, 122 (2019).

[88] R. Blümel, C. Kappler, W. Quint, and H. Walther, Chaos and order of laser-cooled ions in a Paul trap, Phys. Rev. A 40, 808 (1989).

[89] S. Narayanan, N. Daniilidis, S. A. Müller, R. Clark, F. Ziesel, K. Singer, F. Schmidt-Kaler, and H. Häffner, Electric field compensation and sensing with a single ion in a planar trap, J. Appl. Phys. 110, 114909 (2011).

[90] A. Erhard, J. J. Wallman, L. Postler, M. Meth, R. Stricker, E. A. Martinez, P. Schindler, T. Monz, J. Emerson, and R. Blatt, Characterizing large-scale quantum computers via cycle benchmarking, Nat. Commun. 10, 1 (2019).

[91] C. De Grandi and A. Polkovnikov, in Quantum Quenching, Annealing and Computation, edited by A. K. Chandra, A. Das, and B. K. Chakrabarti (Springer, Berlin, Heidelberg, 2010), p. 75. 